\title{
An Estimate of the Bottom Compressional Wave Speed Profile in the Northeastern South China Sea Using "Sources of Opportunity"
}

\author{
Ying-Tsong Lin, James F. Lynch, Senior Member, IEEE, Nick Chotiros, Chi-Fang Chen, Arthur Newhall, \\ Altan Turgut, Steven G. Schock, Ching-Sang Chiu, Louis Bartek, and Char-Shine Liu
}

\begin{abstract}
The inversion of a broad-band "source of opportunity" signal for bottom geoacoustic parameters in the northeastern South China Sea (SCS) is presented, which supplements the towed source and chirp sonar bottom inversions that were performed as part of the Asian Seas International Acoustics Experiment (ASIAEX). This source of opportunity was most likely a "dynamite fishing" signal, which has sufficient low-frequency content $(5-500 \mathrm{~Hz})$ to make it complimentary to the somewhat higher frequency $\mathrm{J}-15-3$ towed source $(50-260 \mathrm{~Hz})$ signals and the much higher frequency $(1-10 \mathrm{kHz})$ chirp signals. This low frequency content will penetrate deeper into the bottom, thus extending the other inverse results. Localization of the source is discussed, using both a horizontal array for azimuthal steering and the "water wave" part of the pulse arrival for distance estimation. A linear broad-band inverse is performed, and three new variants of the broad-band inverse, based on: 1) the Airy phase; 2) the cutoff frequency; and 3) a range-dependent medium are presented. A multilayer model of the bottom compressional wave speed is obtained, and error estimates for this model are shown, both for the range-independent approximation to the waveguide and for the range-dependent waveguide. Directions for future research are discussed.
\end{abstract}

Index Terms-Broadband geoacoustic inversions, shallow water acoustics, South China Sea.

\section{INTRODUCTION}

$\mathbf{O}$ NE of the major objectives of the South China Sea (SCS) component of the Asian Seas International Acoustics Experiment (ASIAEX) was to obtain a good geoacoustic model of the bottom in the experimental area. Having such a model is essential to understanding the propagation and scattering of

Manuscript received August 9, 2003; revised November 14, 2003. This work was supported by the Office of Naval Research under Grant N0 001 498-1-0413, Grant N00014-00-0931, and Grant N00014-01-0772 and by the National Science Council, Taiwan, R.O.C. under Grant NSC92-2611-E-002-005-CCS.

Y.-T. Lin, C.-F. Chen, and C.-S. Liu are with National Taiwan University, Taipei, Taiwan (e-mail: ytlin@whoi.edu).

J. F. Lynch and A. Newhall are with the Woods Hole Oceanographic Institution, Woods Hole, MA 02543 USA (e-mail: jlynch@whoi.edu; anewhall@whoi.edu).

N. Chotiros is with the Applied Research Laboratories, University of Texas, Austin, TX 78712 USA.

A. Turgut is with the Naval Research Laboratory, Washington, DC 02375 USA.

S. G. Schock is with the Florida Atlantic University, Boca Raton, FL 33432 USA.

C.-S. Chiu is with the Naval Post Graduate School, Monterey, CA 93943 USA.

L. Bartek is with the University of North Carolina, Chapel Hill, NC 27514 USA.

Digital Object Identifier 10.1109/JOE.2004.834681 acoustic signals, which was the main focus of the experiment. To obtain a bottom model, three experimental acoustic activities were carried out in ASIAEX: 1) a series of source tows, using a J-15-3 source to transmit signals in the $50-260 \mathrm{~Hz}$ range, to a moored vertical and horizontal array receiver [1];2) chirp sonar surveys along several tracks in the ASIAEX SCS experimental region [2], [3]; and 3) a watergun seismic survey of the overall geology of the ASIAEX SCS site [4]. However, none of these activities was able to provide a low-frequency horizontally propagating signal, such as one obtains from standard "Source of Underwater Sound" (SUS) charge surveys. Thus, the bottom surveys only provided a high-resolution view down to the penetration depth of the $50-\mathrm{Hz}$ sound produced by the J-15-3 source. However, it appears that local "dynamite fishing" activity has provided us with some of the low frequency explosive signals that we were not able to get permission for in the experiment. During the course of the ASIAEX SCS experiment in May 2001, we detected at least four such explosions. Two of the records of these explosions were clipped, as the explosions were obviously nearby and our receiver's dynamic range was set for much lower level signals. Of the remaining two signals, only one had a significant "ground wave" (sometimes called "precursor") component, and we will concentrate on this explosion for this paper. There is probably some further information to be gained from the other signals as well, but we will just choose the most straightforward one for analysis here.

The paper is organized as follows. Section II discusses the general experimental configuration of the ASIAEX experiment, with an emphasis on the experimental components relevant to this present analysis. The general properties of the observed signal, the localization of the source position, and the extraction of the modal group velocity curve are discussed in Section III. Section IV discusses the a priori information that we put into our inverse, specifically the work by Turgut [1], Schock [2], [3], and Bartek and Liu [4]. Section V discusses our inverse methods and results, stressing the compressional wave speed profile in the bottom. The errors inherent in that bottom estimate are shown in Section VI. Last, Section VII presents our final discussion and conclusions.

\section{ASIAEX EXPERIMENT GEOMETRY}

In Fig. 1, we show the acoustic components of the experiment, which are of most interest for the present analyses. The main receiver array for the experiment was the "Shark" mooring 


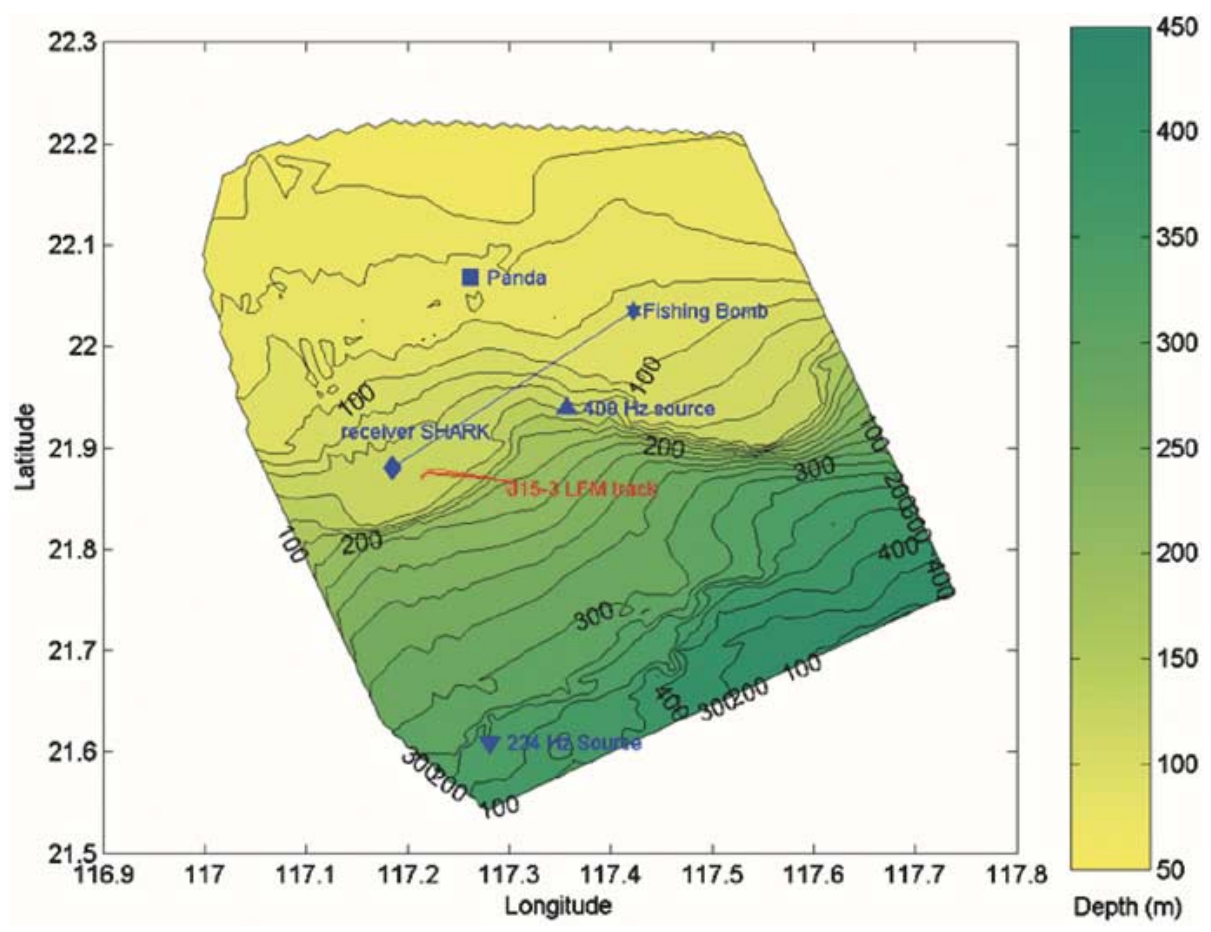

Fig. 1. Locations of the various acoustics sources and receivers of most interest to the 2001 ASIAEX SCS bottom inversion efforts.

(named for the "Shark of Science" insignia painted on the receiver's experimental tow-sled) which was deployed in 124-m water depth. This moored receiver array consisted of a 32-element bottom-lying horizontal line array (HLA) of $\sim 480-\mathrm{m}$ extent (15-m element spacing) and a 16-element vertical line array (VLA) of 82-m extent, with mixed 3.75- and 7.5-m interelement hydrophone spacing [5]. It is in the vicinity of this array that the bottom model we will develop will be most applicable. The PANDA was an inexpensive single-phone receiver that was being tested experimentally as part of ASIAEX [6]. This receiver registered some of the fishing explosions; for these explosions, we can use time delay cross correlation techniques to localize the positions of the sources of opportunity, as the PANDA and Shark are sufficiently separated spatially. Unfortunately, the explosion of most interest in this paper occurred at UTC 1838 on May 16, when the PANDA receiver was already retrieved, so we lost the opportunity for the cross-correlation distance estimate. Turning to the sources, the one of most interest to us is the one farthest to the east in Fig. 1, labeled "Fishing Bomb." The other source of interest is denoted by the red line labeled "J-15-3 LFM track," which shows the tow path that Turgut [1] considered in obtaining his bottom inverse results. There are two other source sites noted in Fig. 1, marked " $224 \mathrm{~Hz}$ source" and " $400 \mathrm{~Hz}$ source." These are the two moored source sites used in the experiment-we need not be concerned with these, except to note that chirp sonar survey lines exist between these source sites and the HLA/VLA receiver.

The J-15-3 tow and fishing bomb locations are both at multikilometer distances from the receiver, and the inverse we derive will represent a range average of the bottom properties between source and receiver. The closest point of approach (CPA) for the J-15-3 source is roughly $3.2 \mathrm{~km}$, with a most distant point of $12.5 \mathrm{~km}$. Moreover, the J-15-3 source to receiver track has a substantial across-shelf component- - there is both a bathymetric and bottom property component to the range dependence. The estimated range for the fishing bomb is about $29.3 \mathrm{~km}$, but with a flatter bathymetric track between source and receiver than the $\mathrm{J}-15-3$ tow line. Range dependence here is still important to the inverse for the bottom, however.

\section{CHARACTERIZATION AND LOCALIZATION OF THE "SOURCE OF OPPORTUNITY" SignAL}

In Fig. 2, we show the time series of the "source of opportunity" explosion, which was received at the VLA/HLA at UTC 1838, May 16, 2001. This signal displays an almost classic form, with a rumbling $\sim 8-\mathrm{Hz}$ "ground wave" early arrival, a sharp explosion "water wave" component following it, and a fairly monochromatic $17.5-\mathrm{Hz}$ "Airy phase" trailing arrival. This explosion has a peak signal-to-noise ratio (SNR) of $\sim 40 \mathrm{~dB}$, which gives us more than adequate power for spectral analysis and other calculations. However, since the exact size of the explosive charge is unknown, we cannot perform exactly calibrated transmission loss calculations and, thus, we cannot estimate medium attenuation. Therefore, our analyses are somewhat restricted as compared to what can be obtained with an explosive source of known level.

Although the time-series representation of the explosion is interesting, the frequency versus time spectral view of the signal is more immediately useful in terms of the broad-band inversion analysis. A spectrogram of the detonation, obtained using standard Fourier techniques, is shown in Fig. 3. To produce this picture, time-dependent frequency analysis was used; specifically, a 4096 sample length fast Fourier transform (FFT) was employed (the array sampling at $3255.208 \mathrm{~Hz}$ ), using a tapered Hann window with a sliding overlap [7]. A 


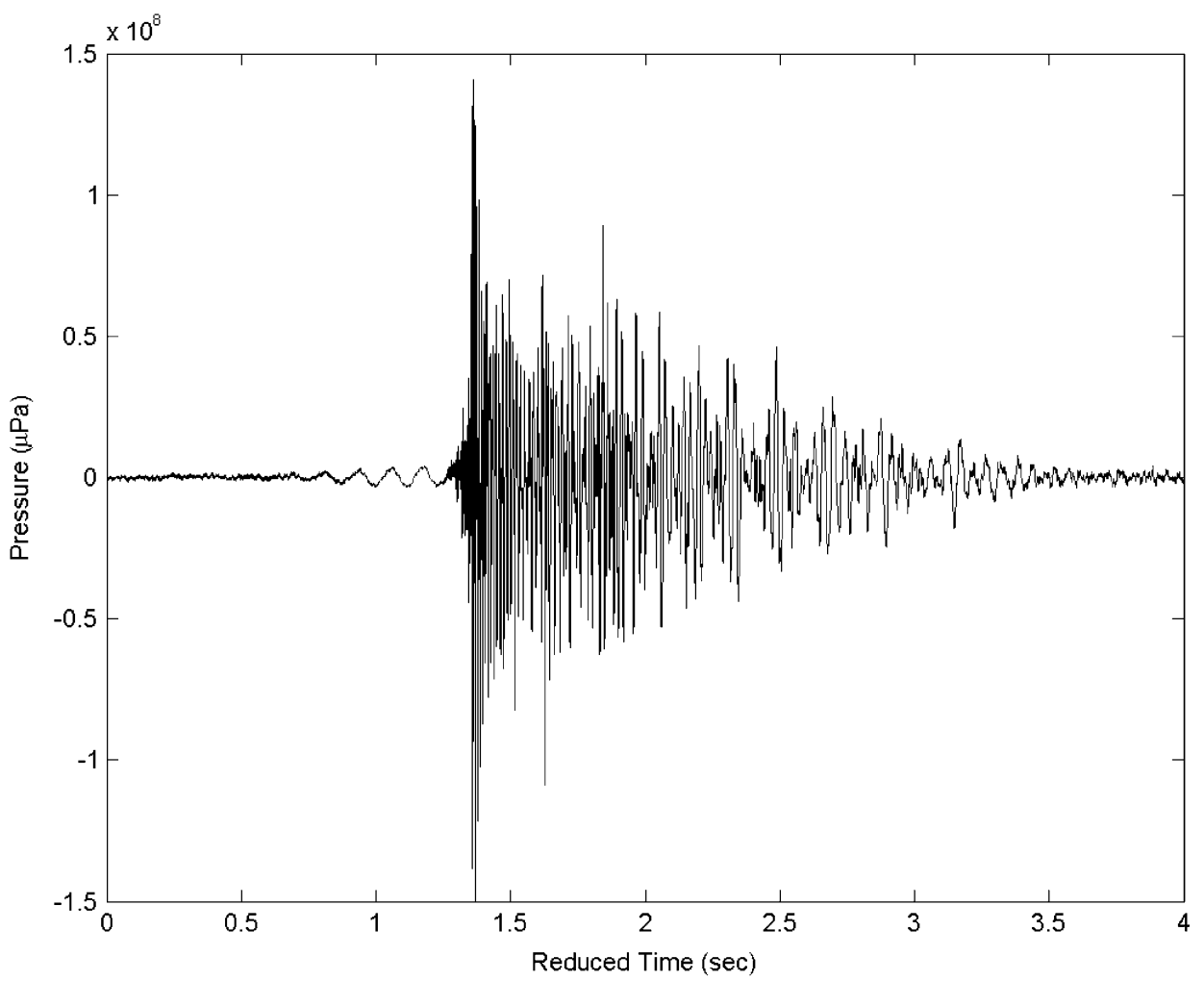

Fig. 2. Explosive "source of opportunity" signal registered at the HLA/VLA receiver on May 16, 2001. The ground wave (quasisinusoidal leading arrival), water wave (large amplitude middle arrivals), and Airy phase (nearly pure tone latest arrival) components are well-defined for this pulse.

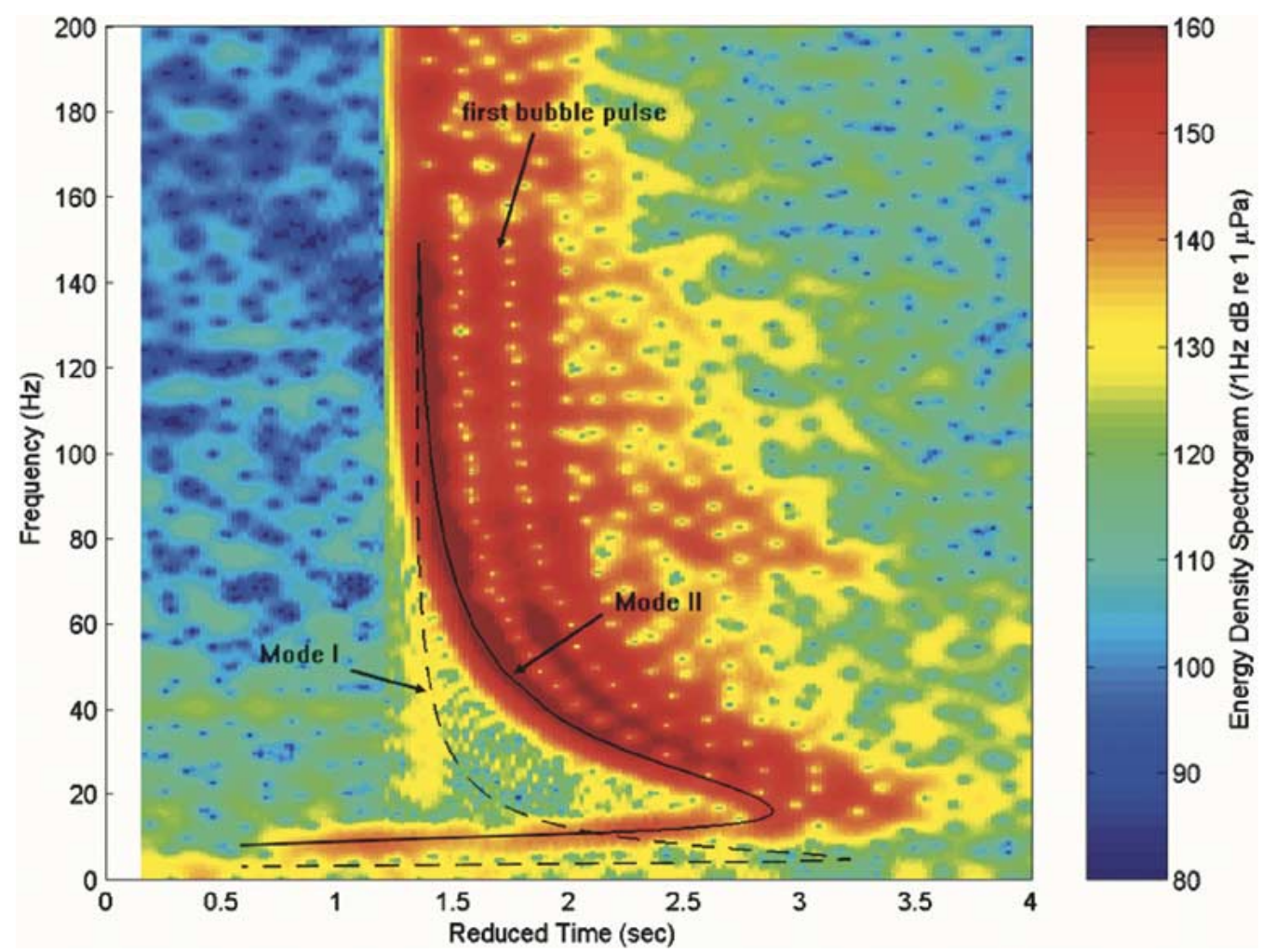

Fig. 3. Spectrogram of the source of opportunity explosion shown in Fig. 2, for a near-bottom hydrophone. The second mode ground wave precursor at $\sim 8$ Hz is clearly seen. One also notes the strong water wave arrival and two subsequent bubble pulses, particularly in the 100-200-Hz range. Mode one is seen faintly, with a weak 4-Hz precursor. The red and blue curves are best fits to the dispersion curves of the first two modes.

wavelet analysis of the signal was also performed; however, the results of this analysis and the traditional spectral analysis were very similar, so we will just present the standard Fourier result here. 


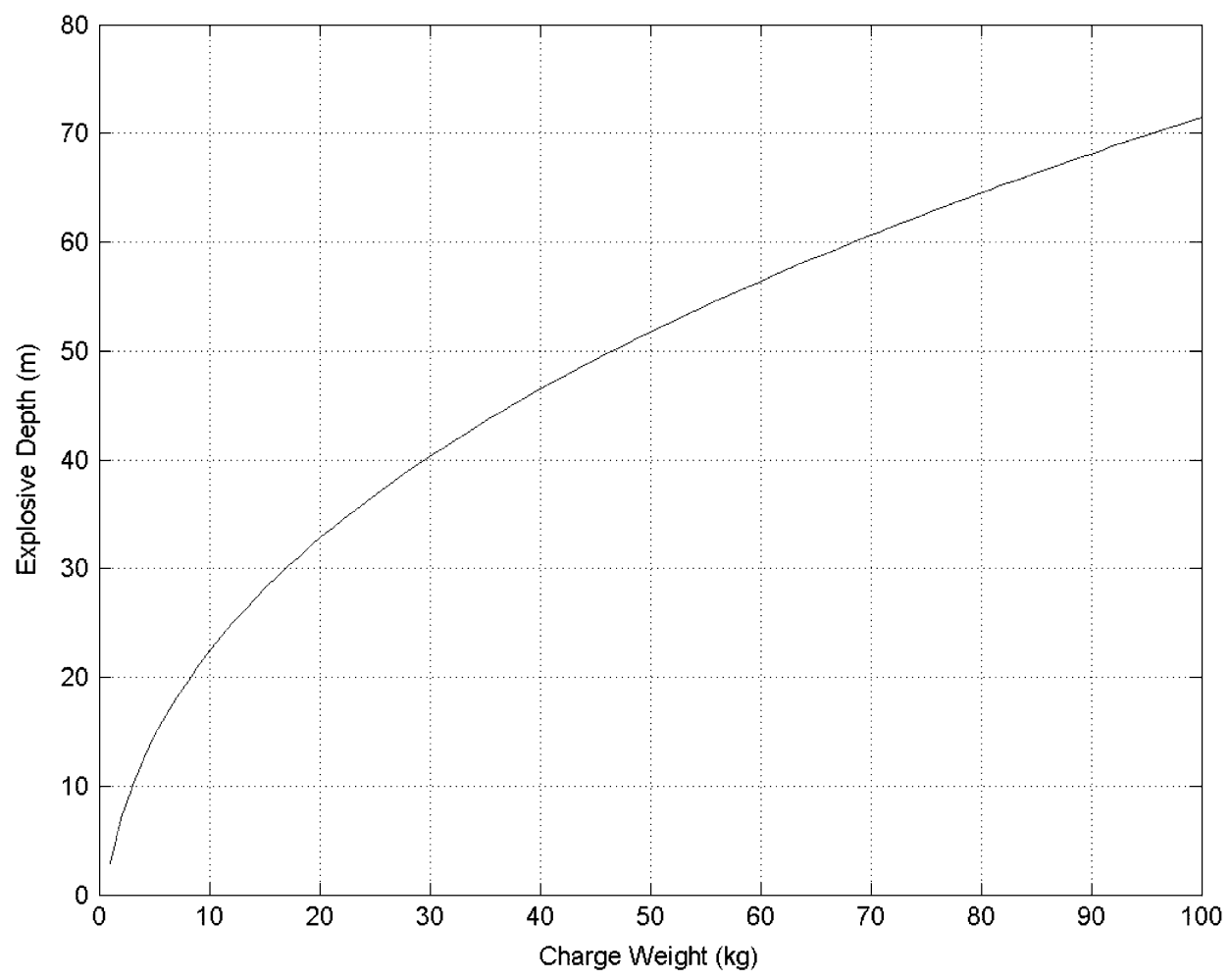

Fig. 4. Depth versus charge weight "ambiguity surface" for an explosive charge in shallow water with a bubble pulse period of $0.25 \mathrm{~s}$.

One of the salient features of the spectrogram in Fig. 3 is the bubble pulses, which are created by alternating cycles of expansion and compression of the hot explosive gases. From the time-frequency analysis, we can see that the time between the bubble pulses is $\sim 0.25 \mathrm{~s}$. This bubble pulse period may be used to estimate the depth/charge-weight ambiguity of the explosion [8] via

$$
T=\frac{K w^{1 / 3}}{(d+10.1)^{5 / 6}}
$$

where $K$ is a proportionality constant and depends slightly on the type of explosive and is equal to 2.11 for TNT, $w$ is the charge weight in kilograms, and $d$ is the depth of detonation below sea surface in meters.Fig. 4 shows that, for any reasonable sized "fishing explosion," which would probably be comprised of a few sticks of dynamite weighing a few kilograms at most, the source depth was probably rather shallow, i.e., less than $20 \mathrm{~m}$ below the surface. This places it in the higher sound speed region near the sea surface (see Fig. 5) for the ASIAEX SCS experimental site. This is important acoustically, in that having a source in a higher speed region favors the excitation of high normal modes versus low normal modes, which explains why we only see mode one faintly in our data, as shown in Fig. 3.

Before we can extract a group velocity dispersion curve from the spectrogram, which is the basic input to our bottom inverse procedure, we need to obtain range and bearing to the source. This will allow us to construct the bathymetry along the track to the source, which is important in a strongly range-dependent environment, such as the ASIAEX SCS site.

Bearing to the explosion was estimated by steering the horizontal line array (HLA) to produce a maximum output at the source bearing. Due to significant near-bottom hydrodynamic stresses imposed by the local oceanography, particularly the nonlinear internal wave field, the bottom-lying horizontal line array was moved and distorted in shape on a routine basis (especially at the tidal period), so we needed to recalibrate the hydrophone positions many times during the experiment. The HLA hydrophone element positions were estimated using three different methods: 1) long baseline navigation using high frequency $(9-13-\mathrm{kHz})$ transponders; 2) using nearby small implosive sources (literally, dropping weighted light bulbs into the water at known locations) [10]; and 3) using the 224-, 400-, $300-$, and $500-\mathrm{Hz}$ moored sources as distant beacons [11]. All these methods produced consistent results, with the last two agreeing the best. A plan view of the hydrophone locations is shown in Fig. 6, for two different dates and using the latter two localization methods. The array is fixed at the origin, due to a heavy anchor, and has some weighting at the far end, but is relatively free to move in the middle. The array on May 16 is almost bow- shaped, which reduces the effective aperture, but also eliminates the left-right ambiguity that a line array typically experiences. By doing simple time delay beamforming on the arrivals and using the element positions shown below, the explosion came from the direction $35^{\circ}$ relative to the east (or $55^{\circ}$ relative to north). The error in this estimate, due to the finite beam width, is on the order of $1^{\circ}$.

The ranging problem is relatively easy if there are two noncolocated receivers available, as we had with the PANDA and HLA/VLA, as seen in Fig. 1. However, in the case we are considering, only the HLA/VLA was available when this explosive pulse was recorded, so we have to rely on another procedure. The procedure we chose was to look at the relative arrival times 


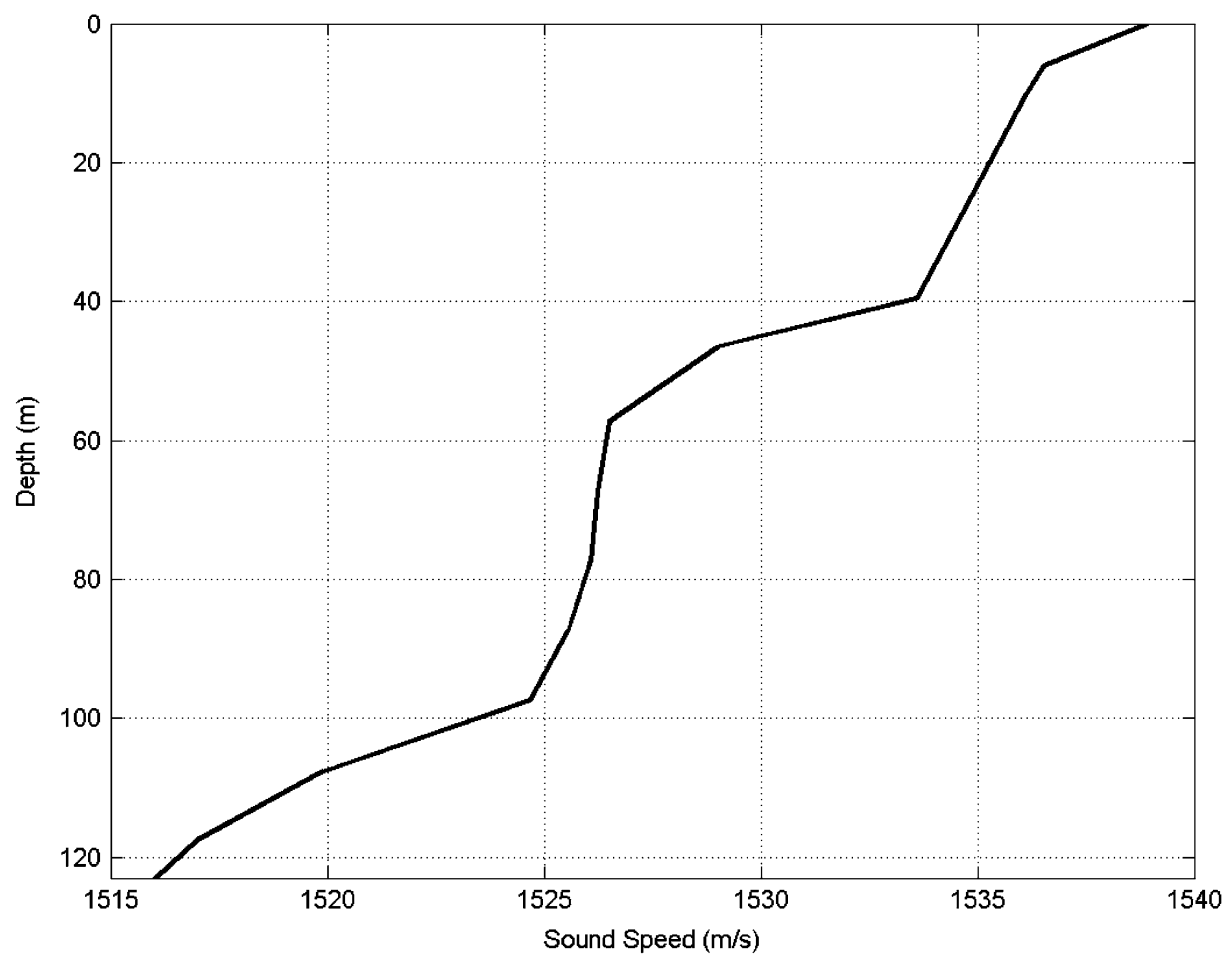

Fig. 5. Sound speed profile obtained from temperature sensors on the VLA at the time the explosion was recorded. Our "source of opportunity" was likely set off at a depth of $\sim 20 \mathrm{~m}$, i.e., in the higher sound speed region of the profile.

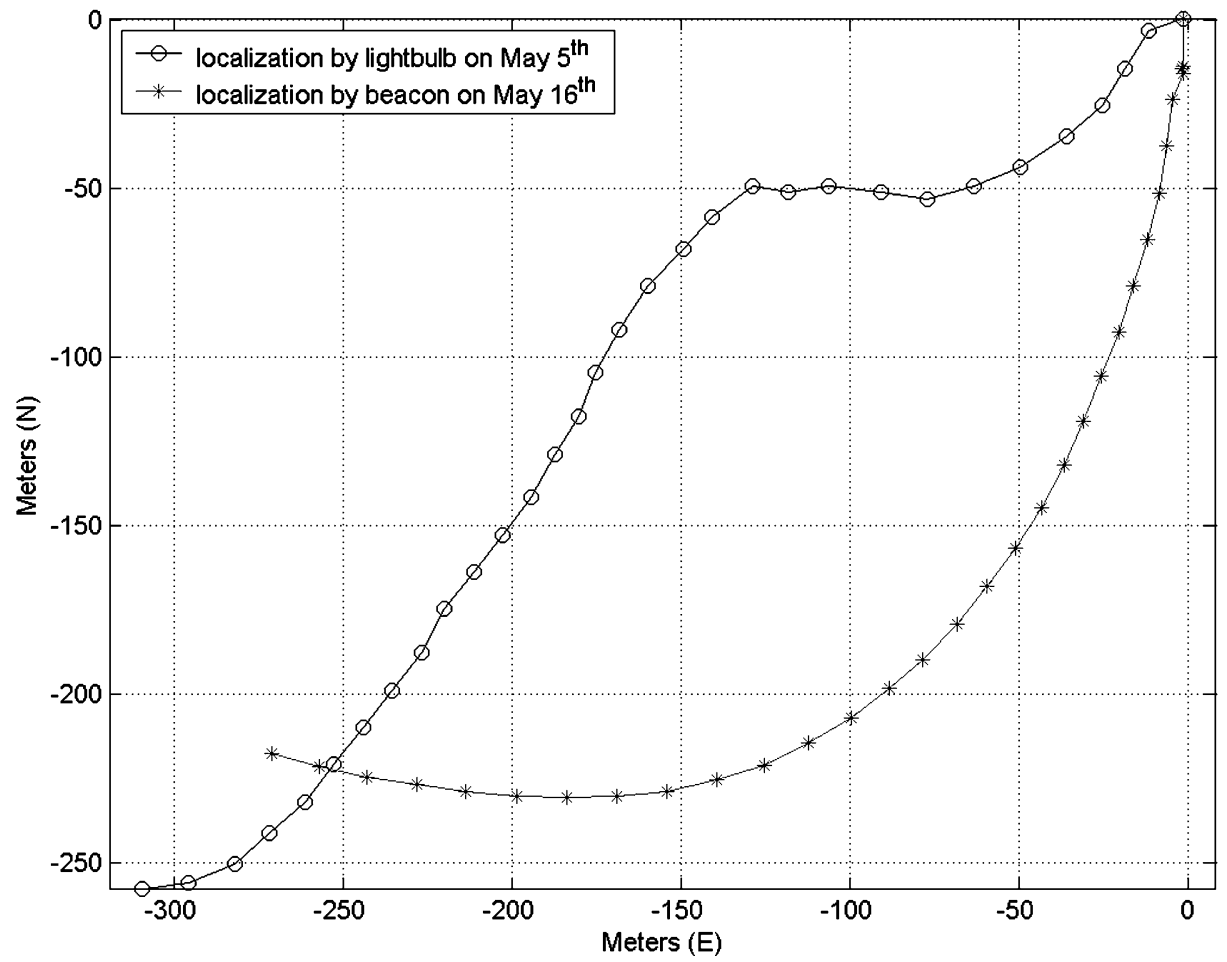

Fig. 6. HLA hydrophone positions on May 5, using implosive source localization and on May 16 (the date of our "fishing bomb source of opportunity"), using long-range moored sources as beacons.

of different frequencies for the same normal mode, which can provide the range. However, in order not to compromise the very low frequency data that we need for the bottom inverse (by using it twice, in effect), we only look at arrival time differences for frequencies greater than $50 \mathrm{~Hz}$ for ranging purposes. There are two reasons for this. First, the inverse by Turgut [1] used
50-200-Hz data to produce a bottom model, so that when we estimate the distance using data from this frequency band, we are doing so using a bottom model which is reasonable. Second, we are more interested in the deep bottom properties in this paper (as Turgut has already inverted for the shallower sediment properties), so that we want to concentrate on the shape of the 


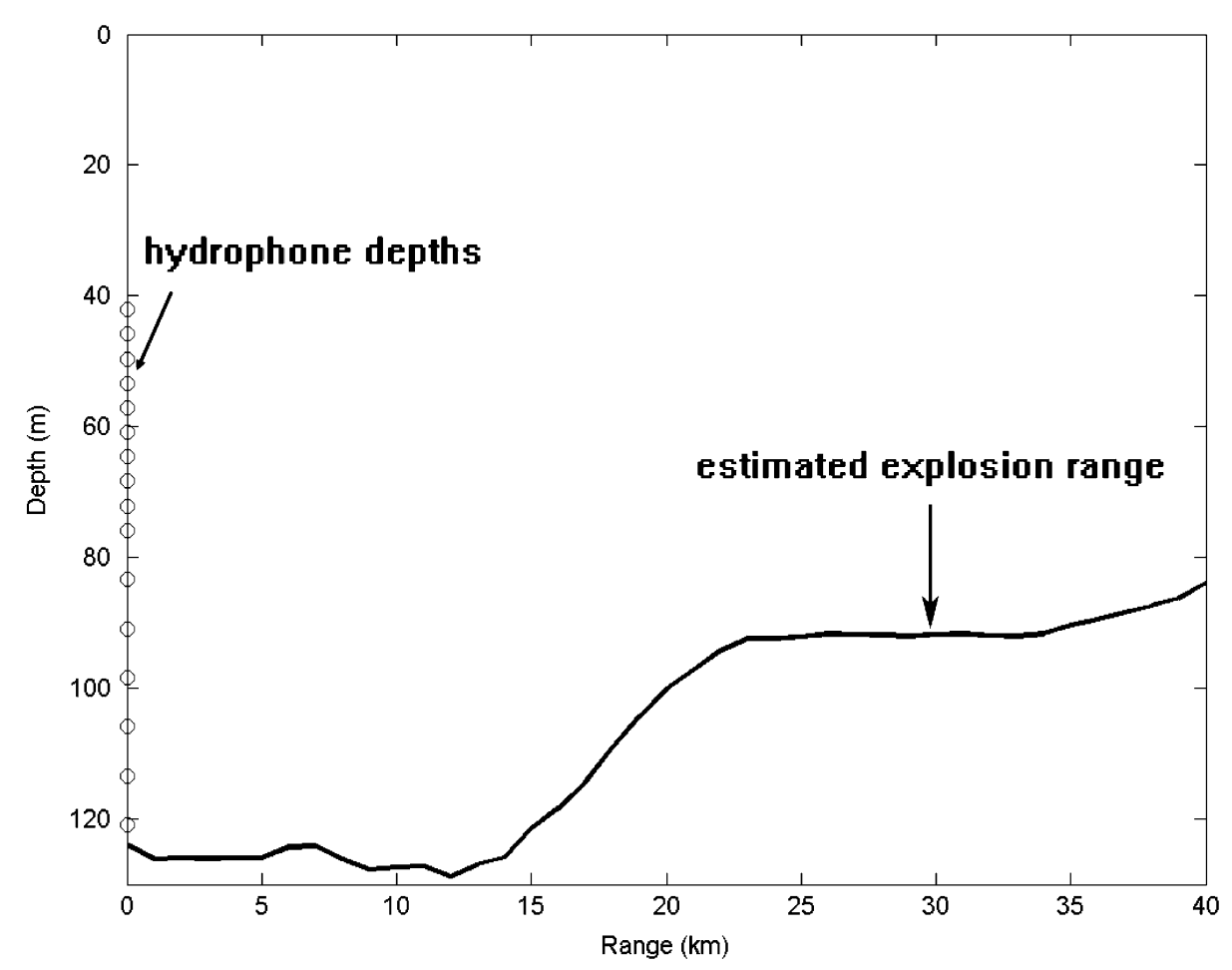

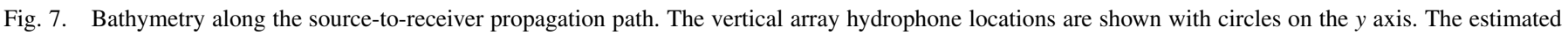
fishing explosion range is indicated by an arrow at a $29.3-\mathrm{km}$ distance from the receiver.

group velocity dispersion curve below $50 \mathrm{~Hz}$. This means that our bottom inverse will mainly comment on the sediment depth region below the turning point of the $50-\mathrm{Hz}$ normal mode.

To calculate the range to the source, we employed the KRAKEN normal mode code [9] to compute "theoretical" adiabatic normal mode group velocity dispersion curves for candidate source ranges. This was done using Turgut's bottom model [1], the water column sound speed profile (Fig. 5) from the experimental site (which was, aside from internal wave activity, spatially and temporally constant for the ASIAEX SCS site), and the bathymetry along the track to the explosion, shown in Fig. 7, using our source-to-receiver bearing estimate. These theoretical curves were generated for a large variety of range possibilities and then a best fit to the data, shown in Fig. 3, was obtained. In Fig. 8, we show the best fit comparison between the theoretical curves and the data, which results in a range of $29.3 \mathrm{~km}$ for the explosion. This estimate has error inherent in it due to errors in the bottom model, SNR, etc. We will quantify that error in a later portion of this paper, where we discuss the error in the inversion for the bottom.

We should also discuss our claim that the strongest mode signal, which we are using as our prime bottom inverse data, is mode two rather than mode one, which is what one usually expects to see. This claim is supported by the facts that: 1) vertical array mode filtering (using the cleaner frequencies in the dispersion curve) clearly shows the mode two shape (Fig. 9); 2) the strongest mode's cutoff is at $8 \mathrm{~Hz}$, indicative of mode two, whereas only a very weak signal at the lowest hydrophones is seen at $4 \mathrm{~Hz}$, indicative of mode one (Fig. 3); and 3) using mode one in the source ranging estimate gives an unreasonable result of hundreds of kilometers.
In fact, we deemed the mode one signal too weak to use for the inversion process in this paper. Normally, we use all the modes and frequencies available to improve the inverse result. Physically, we believe that the faintness of mode one is due to its weak excitation at the (shallow) source. The absence of higher modes in the data (three and higher) is probably due to their higher attenuation, leading to mode stripping over the $\sim 29-\mathrm{km}$ path.

\section{A PRIORI BotTOM GeOACOUSTIC INFORMATION, GROUP VELOCITY DISPERSION CURVES, AND THE CONSTRUCTION OF A BACKGROUND MODEL FOR THE BROADBAND INVERSION}

In this section, we will discuss three items: 1) the bottom model created by Turgut based on the J-15-3 and chirp sonar data; 2) the creation of group velocity dispersion curves using the fishing bomb data and Turgut's model; and 3) extending Turgut's bottom model with the fishing bomb data to get an improved background model to use for linear perturbative inverse methods.

\section{A. Turgut's Bottom Model}

There already is a significant amount of information about the bottom available from other measurements made during the ASIAEX SCS experiment. The two that most interest us here are the chirp sonar images, obtained by Schock, and the J-15-3 towed source bottom inversions, performed by Turgut [1]-[3].

A cross-shelf transect image (Fig. 10) made by the chirp sonar shows the extreme cross-shelf variability, both in the bathymetry and in the subbottom stratification. The portion of the image of most interest to us is the shallow portion [left-hand side (LHS) of the image], where the acoustic receiver array is located. In 


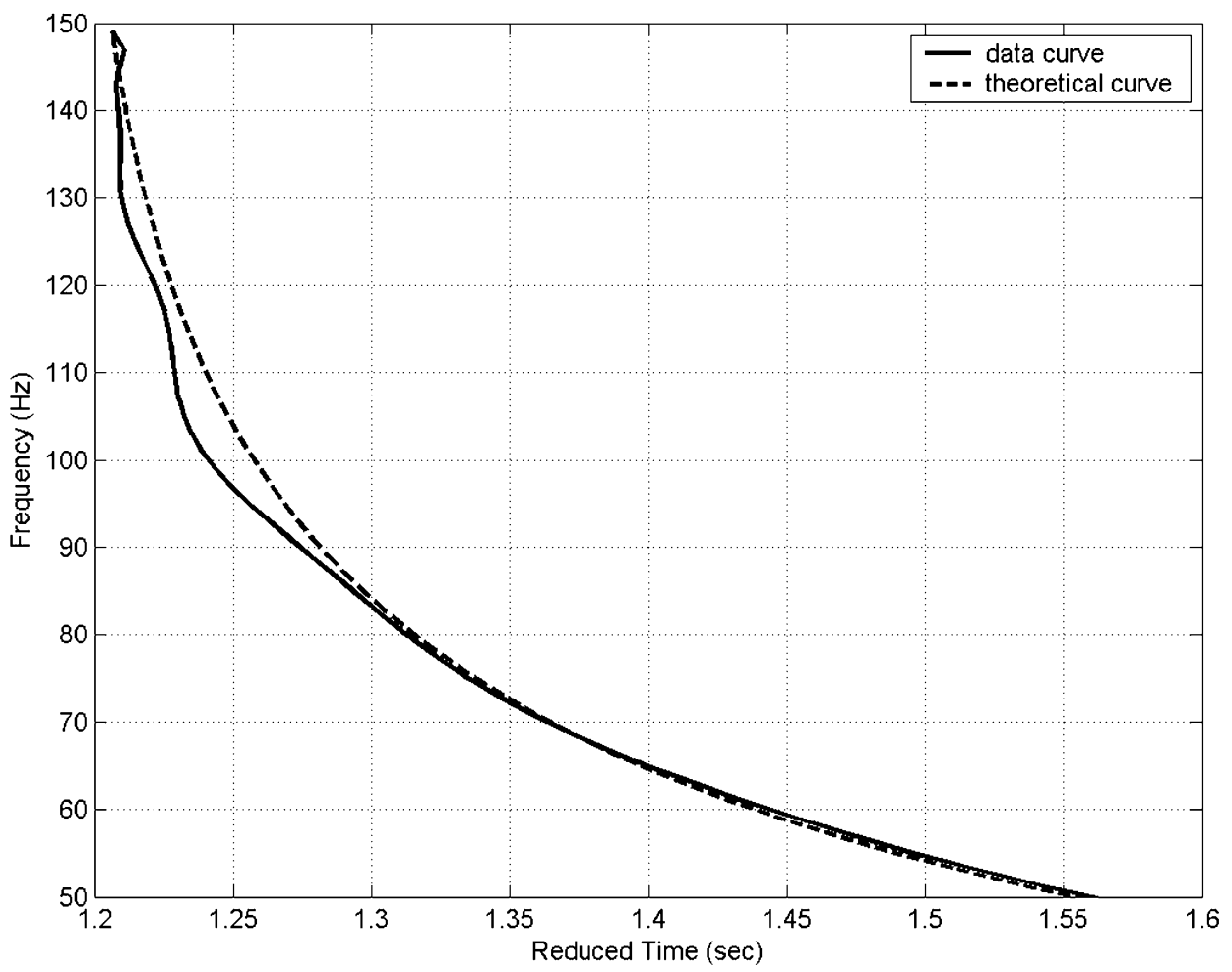

Fig. 8. Best fit of the theoretical dispersion curve to the data from Fig. 3 over the $50-150-\mathrm{Hz}$ frequency band. This fit was obtained using a distance of $29.3 \mathrm{~km}$, which we take as our nominal distance to the explosion.

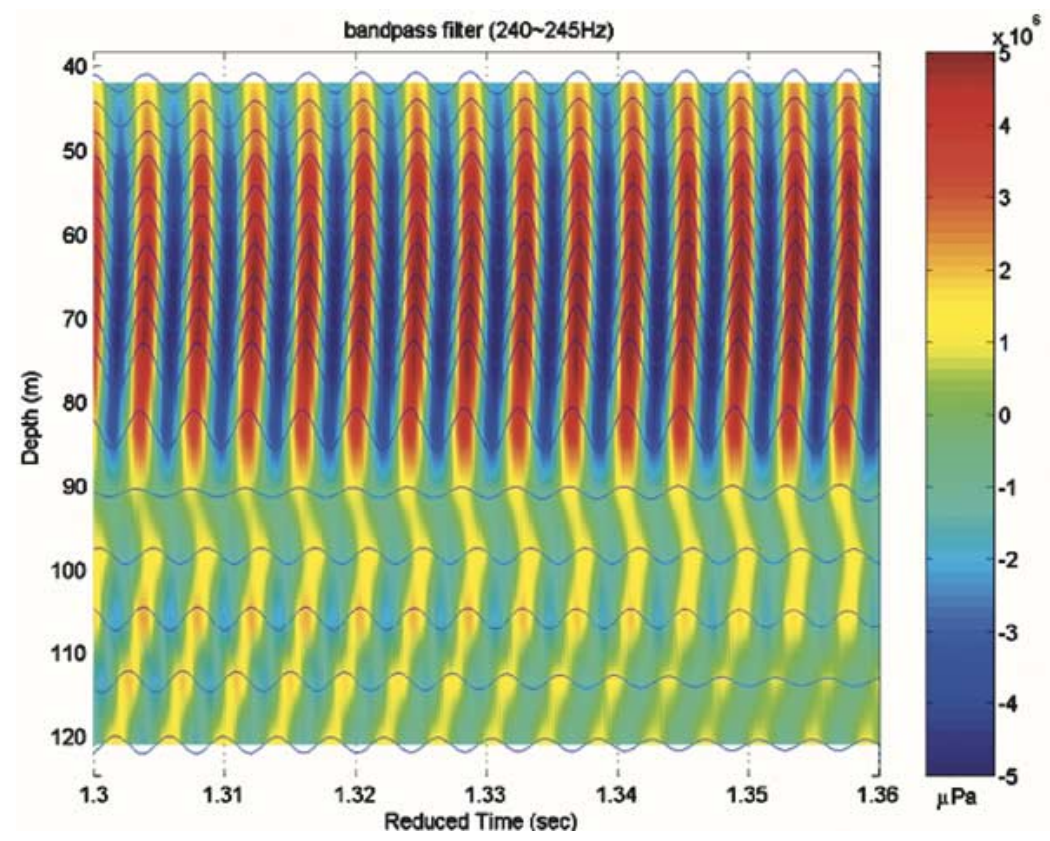

Fig. 9. Mode filtered vertical array data showing mode 2.

this part of the image, there is a (roughly) 10-m-thick layer of near surface sediment overlying a deeper homogeneous layer. These are tentatively identified as a silty sand layer overlying a sandy "half-space."

Using this chirp sonar layering information as input to his towed source inverse, Turgut [1] created the bottom model shown in Table I. In this inverse, the values of the density in the two layers are generated from Hamilton's regression equations [12], [13].

\section{B. Creation of Group Velocity Dispersion Curves}

The second mode is transmitted through the range-dependent environment with range-dependent group velocity. Since we now have a distance estimate to the explosion, we can create the range-independent and range-dependent group velocity dispersion curves for this mode from the time-frequency diagrams we have considered. The equation below is for the range-averaged case (the range-independent case is obtained in sim- 


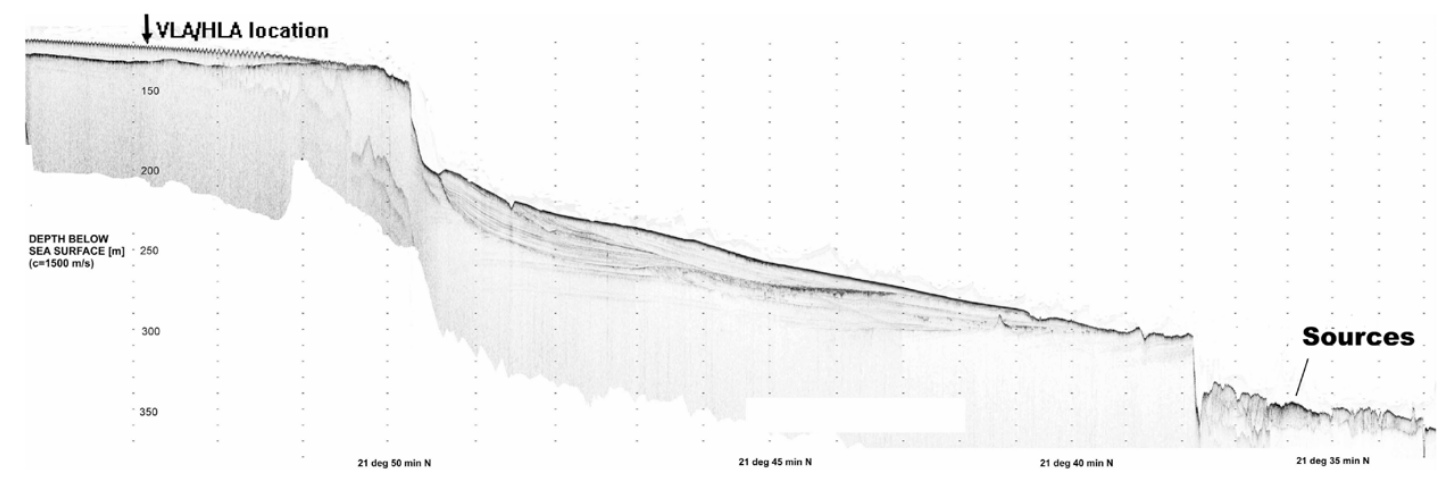

Fig. 10. Across-shelf chirp sonar image of the bottom at the ASIAEX SCS site. The HLA/VLA is located at the 124-m isobath along this transect, marked by a dark arrow.

TABLE I

Sediment Geoacoustic Model Created From J-15-3 Tow Data

\begin{tabular}{l|l}
\hline Source frequency range $(\mathrm{Hz})$ & $50-200$ \\
\hline Sound speed in layer of $10 \mathrm{~m}$ thickness $(\mathrm{m} / \mathrm{s})$ & $1603.16 \pm 16.90$ \\
\hline Sound speed in half space $(\mathrm{m} / \mathrm{s})$ & $1697.32 \pm 42.19$ \\
\hline Attenuation $(\mathrm{dB} / \lambda)$ & $0.778 \pm 0.149$ \\
\hline Density in layer of $10 \mathrm{~m}$ thickness $\left(\mathrm{g} / \mathrm{cm}^{3}\right)$ & $1.626 \pm 0.170$ \\
\hline Density in the half space $\left(\mathrm{g} / \mathrm{cm}^{3}\right)$ & $1.740 \pm 0.142$ \\
\hline
\end{tabular}

ilar fashion). We can get the range-averaged modal group velocity from the travel time $T$, which is estimated from the measured $T_{\text {relative }}$ and the theoretical group velocity at $150 \mathrm{~Hz}$ using Turgut's bottom model. Specifically

$$
\bar{V}_{G 2}(\omega)=\frac{R}{T}=\frac{R}{T_{\text {relative }}+R / \bar{V}_{G 2}\left(\omega_{0}\right)}
$$

where $R$ is the range estimate, $T$ is the travel time, $T_{\text {relative }}$ is the relative arrival time, and $\bar{V}_{G 2}\left(\omega_{0}\right)$ is the theoretical modal group velocity at frequency $\omega_{0}$ (i.e., $150 \mathrm{~Hz}$ in our analysis) using Turgut's model, which is calculated from

$$
\frac{1}{\bar{V}_{G 2}\left(\omega_{0}\right)}=\frac{1}{R} \int_{0}^{R} \frac{d r}{V_{G 2}\left(\omega_{0}, r\right)} .
$$

In the above formulation, the modal group velocity at $\omega_{0}, v^{G}\left(\omega_{0}\right)$ is estimated using the in situ sound speed in water, Turgut's bottom model, and either a flat bathymetry of 124-m depth (the range-independent case) or the actual bathymetry (the range-dependent case). We obtain the group velocity curves shown in Fig. 11 and note that the group velocity curves for the range-independent and range-dependent cases are significantly different, indicating that the bottom model generated might also be different. We now return to estimating our bottom background model.

\section{Extended Bottom Background Model}

We now discuss estimating the bottom model using the explosive signal, which provides lower frequency content and allows us to complement Turgut's bottom model. First, we notice that, for Turgut's bottom model, $50-\mathrm{Hz}$ energy penetrates to about 50-m depth so that, using frequencies below $50 \mathrm{~Hz}$, we are justified in adjusting the sound speed below $50 \mathrm{~m}$ to values that differ from Turgut's "half space." We may also adjust values above $50 \mathrm{~m}$ based on our low-frequency data, but should note that we need to keep that part of the bottom model close to Turgut's result, so that our inverse remains consistent with both the higher frequency J-15-3 data and the lower frequency "source of opportunity" data.

To extend Turgut's result, we find that we need to add two more layers below 50-m depth to the sediment model. One is the halfspace basement and the other is a layer lying between the basement and those upper two layers that we adopt from Turgut's bottom model. We assume that the interfaces between each layer are parallel with the sea floor and the depth dependency of bottom properties relative to the sea floor is range independent.

In fact, the resolved bottom model in the range-dependent case is range averaged. Since we have only one source station for data, the range variability of the sea bottom cannot be resolved. Furthermore, a linear perturbative formulation for range-averaged modal group velocity in Appendix B also shows the feasibility to invert a range-average model from range-averaged data.

To get the two new layers, let us first look at the procedure for the calculation of the range for the range-independent case. (For the range-dependent case, the same procedure is followed.) We first consider the "basement" layer, the sound speed of which is determined by the observed cutoff frequency of mode two. Since we know the horizontal wavenumber at $150 \mathrm{~Hz}$ from Turgut's bottom model, we can integrate the inverse of the group velocity curve from 150 to $8 \mathrm{~Hz}$ (the cutoff frequency) to get the horizontal wavenumber at $8 \mathrm{~Hz}$. The value of the integration obtained is $k_{r}(8 \mathrm{~Hz}) \sim 2.039 \times 10^{-2}$ $\mathrm{m}^{-1}$. The sound speed in the bottom halfspace thus should be $\mathrm{c}=\omega / k_{r}$, which is $2340 \mathrm{~m} / \mathrm{s}$.

However, just putting this halfspace basement directly under Turgut's original model at 50-m depth does not give a reasonable model to fit our group velocity data. Thus, one more layer is added. As regards the thickness and sound speed of this new 


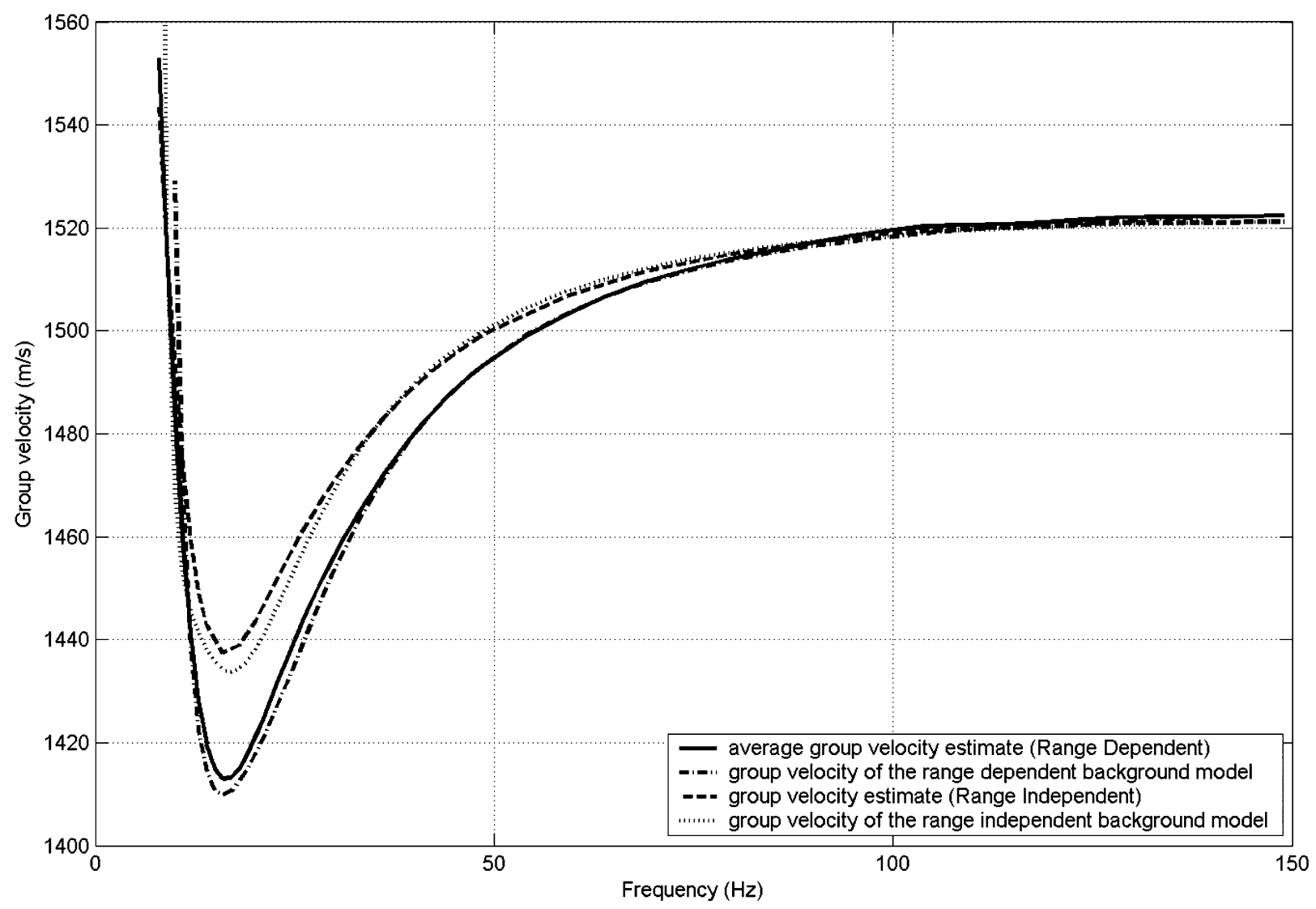

Fig. 11. Group velocity of measured data versus that generated by the background model, for both range-independent (receiver depth only) and range-dependent (true) bathymetry.

layer, we discover an extremely significant fact-the Airy phase frequency is rather sensitive to these two parameters. Consequently, we adjust them to give the correct theoretical position of the Airy phase by simple iteration of forward models. The result shows a $45-\mathrm{m}$ - thick layer with sound speed of order of $1850 \mathrm{~m} / \mathrm{s}$. Although the Airy phase plays a dominant role to determine the thickness and sound speed in this layer, the rest of the low-frequency signal, whose energy penetrates the whole depth, is also helpful in constraining the new layer, as the Airy phase frequency for one mode is a single datum and, thus, cannot unambiguously constrain two layer parameters (the thickness and sound speed).

Our low-frequency data also allows us to modify the sound speed in the upper two layers above $50 \mathrm{~m}$, but it should be noted that the sound speed in these two layers still must be close to Turgut's result, so that our inverse remains consistent with both the higher frequency data and the lower frequency data. Therefore, we slightly adjust the sound speed in layer II to $1640-1660 \mathrm{~m} / \mathrm{s}$, which is still within the error bound of Turgut's results (see Fig. 12), so as to improve the mean-square fit of theoretical the modal group velocities below $50 \mathrm{~Hz}$ to the data. Finally, we add a slight sound speed gradient to layer III $(1800-1900 \mathrm{~m} / \mathrm{s})$ in accord with Hamilton's regressions [13], to compensate for the overburden pressure (weight of the sediments above). The model thus generated is shown in Fig. 12 and the corresponding theoretical modal group velocity of mode two is shown in Fig. 11.
There are three things that we should note from Figs. 11 and 12. First, we can see from Fig. 11 that the (range-independent and range-dependent) bottom background models we generated already provide good fits to the group velocity dispersion curve data. Second, we see from Fig. 12 that our bottom background model still agrees rather well with Turguts model in the top $50 \mathrm{~m}$ of the sediment, as we claimed it should. And third, we see that the layers below $50 \mathrm{~m}$ are different between the range-independent and range-dependent cases. In particular, the range-dependent case shows a slightly faster bottom sound speed.

\section{INVERSION FOR BOTTOM PROPERTIES}

With a reasonable background model(s) and the group velocity data in hand, we now can proceed with the linear broadband inverse for the bottom. We will look first at the range-independent case. The formalism we will use was developed by Rajan and Lynch [14], [15] and has been successfully applied to a number of data sets. Specifically, this method uses the measured (reciprocal) perturbation of the group velocity from the assumed background model to infer the perturbation of sound speed from the background model. This approach gives an integral equation

$$
\begin{aligned}
\left.\left(\frac{1}{v_{n}^{G}}-\frac{1}{v_{n}^{G(0)}}\right)\right|_{\omega} & =\frac{-1}{k_{n}^{(0)}} \int_{0}^{\infty} \rho^{(0)-1}\left[\left(2 \omega^{-1}-\frac{1}{k_{n}^{(0)} v_{n}^{G(0)}}\right)\right. \\
& \left.\times\left|Z_{n}^{(0)}\right|^{2}+\frac{d}{d \omega}\left|Z_{n}^{(0)}\right|^{2}\right] \frac{\omega^{2}}{c^{(0) 3}} \Delta c d z
\end{aligned}
$$




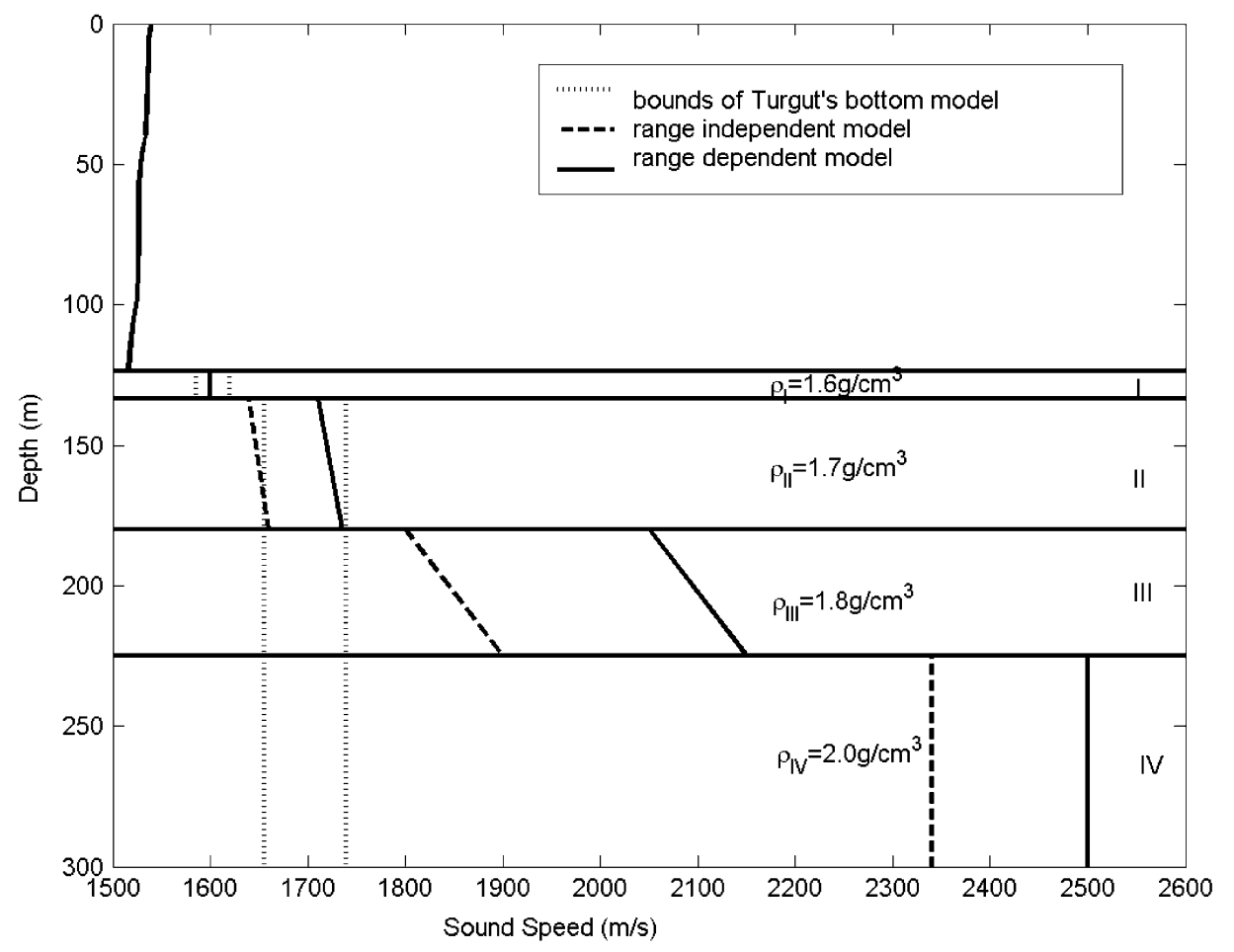

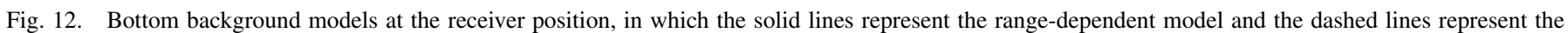

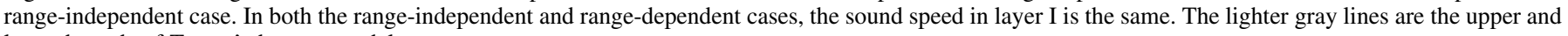
lower bounds of Turgut's bottom model.

where $Z_{n}^{(0)}, k_{n}^{(0)}$ and $v_{n}^{G(0)}$ are the mode function, modal eigenvalue (horizontal wavenumber), and the modal group velocity of the $n$th mode in the background model. This equation is a Fredholm integral equation of the first kind and is amenable to a variety of inverse solutions. A common formulation is to reduce this equation to a linear equation using a numerical quadrature scheme to represent the integral. Thus, we obtain the matrix form

$$
\mathbf{d}=\mathbf{G q}
$$

where $\mathbf{d}$ is the $\mathrm{N} \times 1$ data vector (the perturbed group velocity at $\mathrm{N}$ frequencies), $\mathrm{G}$ is the $\mathrm{N} \times \mathrm{M}$ kernel matrix of the perturbation integral equation, and $q$ is the $M \times 1$ solution vector (an M layer bottom model perturbation). Using the frequencies below $50 \mathrm{~Hz}$ as data and performing the inverse, we find, rather interestingly, that our background model is not significantly improved by the procedure. Indeed, the $\Delta \mathrm{c}$ produced just seems to add noise to the background model and do not improve the mean-square fit of the group velocity model to the group velocity measurement, shown in Fig. 11. Thus, it seems that we started with a very good model to begin with. In a sense, this is not a surprise, because we initially adjusted two of the most important and sensitive pieces of low-frequency data, the cutoff frequency and the Airy phase, to give the "background" bottom model. In fact, this was done in part using two new perturbative inverses based on those quantities, which we describe in Appendix A. We also adjusted the sound speed of layer number III (Fig. 12) iteratively beforehand, which is basically a nonlinear inverse procedure. Since the linear inverse does not improve our background model, we can thus say that, to the accuracy of the linear method, we have obtained a reasonable range-independent bottom model.

For the range-dependent inverse problem, we present an extension of the Rajan-Lynch formalism (Appendix B), which uses the range averaged group velocity rather than the range-independent group velocity as input to the inversion process. This extension is an approximation and not exact-the error associated with it is also discussed in Appendix B. Applying this formalism, we obtained the same type of result as in the range-independent case. Due to the initial estimate of the background model again being very good, the linear inverse does not significantly improve the bottom model, so we deem our initial guess to be an adequate one.

\section{ERROR ESTIMATES FOR THE BOTTOM MODEL}

Although our initial background models were sufficiently good, the work done in creating the linear inverse estimates is far from wasted, in that one still needs the kernel of that inverse to determine the resolution and variance of the bottom models generated. Looking first at the vertical resolution of the estimate, we have [14], [15] for the resolution kernel $R$ that

$$
R\left(z, z_{0}\right)=G^{T}\left(z_{0}\right)\left(G G^{T}\right)^{-1} G(z)
$$

where

$$
\begin{aligned}
\left(G G^{T}\right)_{i j} & =\int_{0}^{a} G_{i}(z) G_{j}(z) d z \\
G\left(z_{0}\right) & =\left[G_{1}\left(z_{0}\right), \ldots, G_{N}\left(z_{0}\right)\right] .
\end{aligned}
$$

We can reduce this depth-dependent function of $z$ into a single number, the "resolution length," at any given $z$ simply by reporting the width of $R\left(z, z_{0}\right)$. We do that in these analyses, 
TABLE II

Estimates Of the EXPlosive Source RANGE AND Relative ARrival Time AND Their ERrors

\begin{tabular}{c|c|c}
\hline & $\begin{array}{c}\text { source range } \\
\text { range-independent/range-dependent }\end{array}$ & relative arrival time \\
\hline Estimate & $38.9 \mathrm{~km} / 29.3 \mathrm{~km}$ & see Figure 3 \\
\hline $\begin{array}{c}\text { error range } \\
\text { (standard deviation } \sigma)\end{array}$ & $\pm 2.0 \mathrm{~km} / \pm 0.852 \mathrm{~km}$ & $\pm 1.25 \times 10^{-2} \mathrm{sec}$ \\
\hline
\end{tabular}

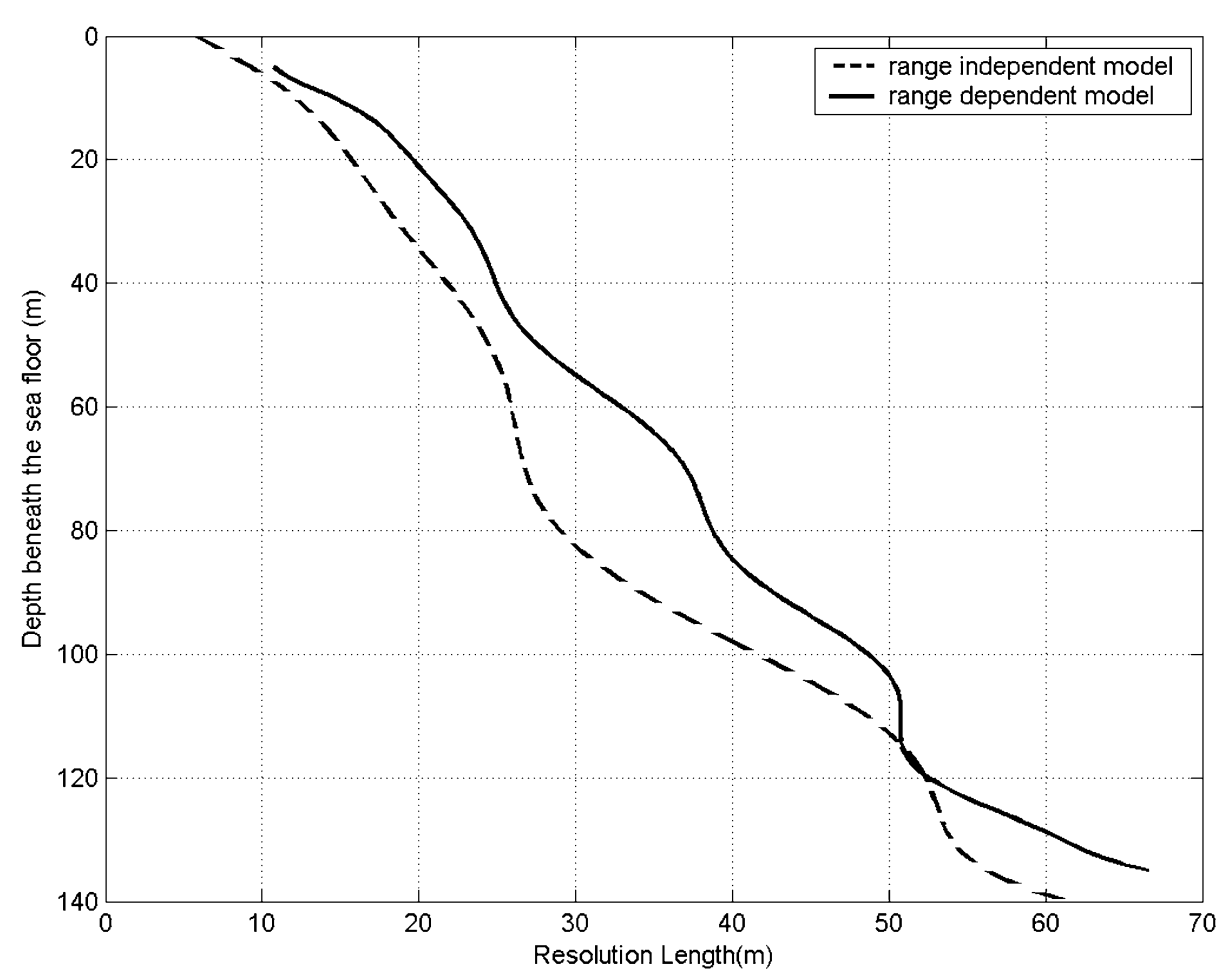

Fig. 13. Resolution length versus depth for the range-independent bottom estimate (the dashed line) and the range-dependent bottom estimate (solid line).

obtaining the results shown in Fig. 13. This resolution length physically represents how much of a "vertical average" of the medium properties the inverse produces at any given depth. We should note that this kernel just represents the resolution obtained from our broad-band modal inverse; the improved resolution one gains from combining the chirp sonar, the J-15-3 tow source inverse, and the broad-band source of opportunity inverse is not reported here, as the kernel for that combined inverse is much more difficult to construct.

To calculate the variance in the estimate, we again adopt the methods used by Rajan, Lynch et al. [14], [15]. Assuming that the errors in each data point are uncorrelated and that the error variance is the same for all data (useful assumptions, though not always correct) we obtain the variance $\mathrm{Cov}_{q}$ from

$$
\operatorname{Cov}_{q}=\sigma_{d}^{2}\left(\mathbf{a}^{T} \mathbf{a}\right)
$$

where $\mathbf{a}$ is the generalized inverse matrix of $\mathbf{G}$ (3). However, if the error variance is not the same for all data (i.e., frequency dependency in our case), the variance $\operatorname{Cov}_{q}$ becomes

$$
\operatorname{Cov}_{q}=\mathbf{a}^{T} \operatorname{Cov}_{d} \mathbf{a},
$$

where $\operatorname{Cov}_{d}$ is the covariance matrix of the data error. If the errors in each data point are uncorrelated, this matrix is a diagonal matrix containing the error variance.

We constructed the generalized inverse via the singular value decomposition and neglected the small singular values, which can cause huge variations [16]. For this paper, there are only four singular values considered. This leaves only the variance $\sigma_{d}^{2}$ to determine in the data $v_{g}^{-1}$.

The variance of the modal group velocity in the range-dependent case is determined as the following from the theory of the propagation of error [17]:

$$
\begin{aligned}
\left(\frac{\sigma_{v^{G}}}{v^{G}}\right)^{2}=\left(\frac{T_{\text {relative }}}{T}\right)^{2} & \left(\frac{\sigma_{R}}{R}\right)^{2}+\left(\frac{\sigma_{T_{\text {relative }}}}{T}\right)^{2} \\
& +\left(\frac{v^{G}}{v^{G}\left(\omega_{0}\right)}\right)^{2}\left(\frac{\sigma_{v^{G}\left(\omega_{0}\right)}}{v^{G}\left(\omega_{0}\right)}\right)^{2} .
\end{aligned}
$$

In (7), the meaning of each of the terms on the right-hand side (RHS) is the same as in (1), i.e., the errors are in the range of explosive source range, the relative arrival time, and the group velocity at $150 \mathrm{~Hz}$ (listed in Table II). The last term on the RHS is found to be much smaller than the first terms, since we have Turgut's bottom model, which is adequate for modeling sound 


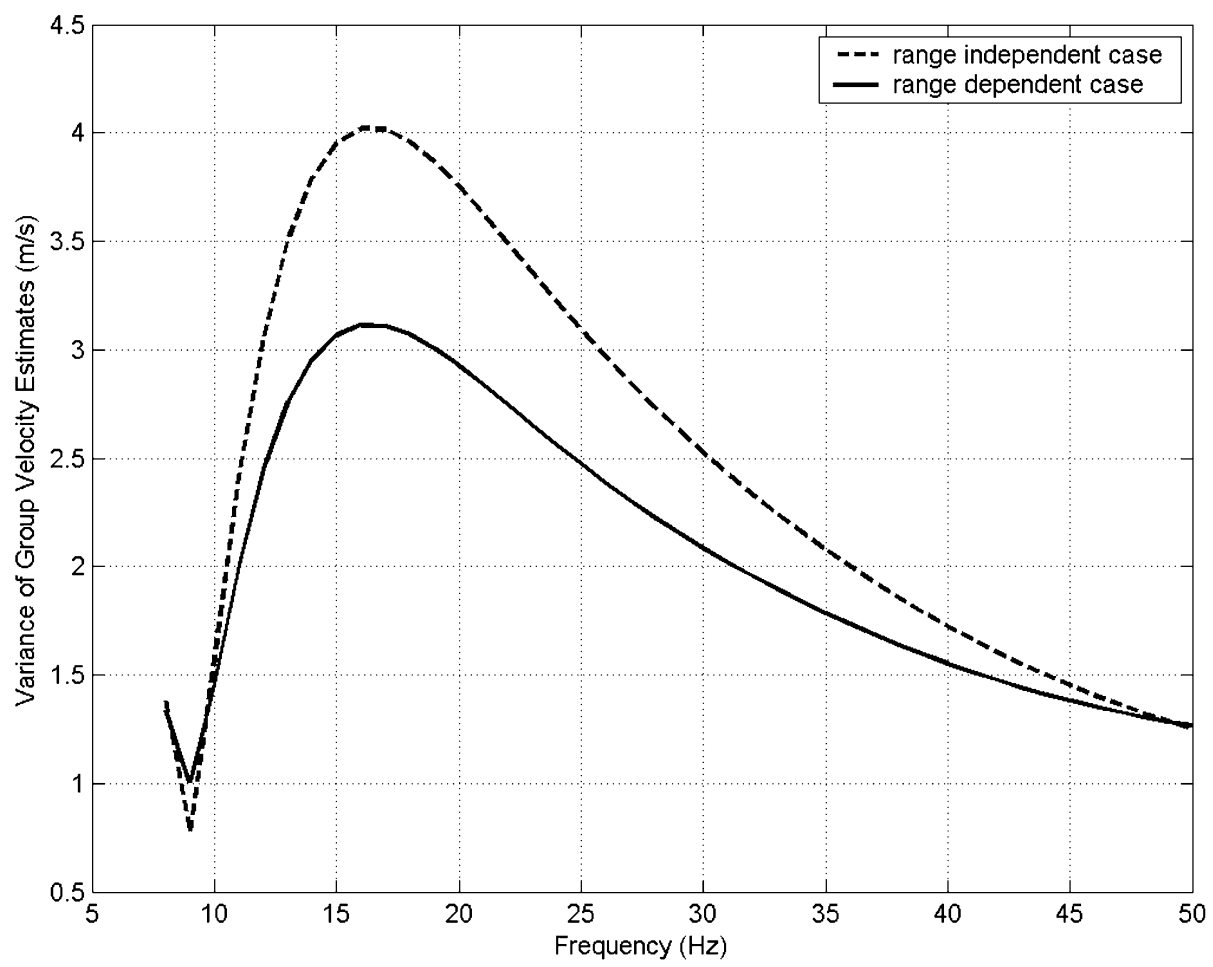

Fig. 14. Frequency-dependent variance of modal group velocity estimates in both cases. In the range-dependent case, the modal group velocity estimate is a range-average velocity.

propagation at this frequency. Hence, the variance of the modal group velocity can be approximated by

$$
\sigma_{v^{G}}^{2} \approx\left(v^{G} \frac{T_{\text {relative }}}{T}\right)^{2}\left(\frac{\sigma_{R}}{R}\right)^{2}+\left(v^{G} \frac{\sigma_{T_{\text {relative }}}}{T}\right)^{2} .
$$

The variance of the range-averaged modal group velocity in the range-dependent case is approximated by

$$
\sigma_{\bar{v}^{G}}^{2} \approx\left(\bar{v}^{G}-\frac{\bar{v}^{G 2}}{v^{G}\left(\omega_{0}, R\right)}\right)^{2}\left(\frac{\sigma_{R}}{R}\right)^{2}+\left(\bar{v}^{G} \frac{\sigma_{T_{\text {relative }}}}{T}\right)^{2} .
$$

Both of these two terms are frequency dependent, as shown in Fig. 14.

Some further discussion of the way we obtained standard deviation $\sigma_{\mathrm{R}}$ in Table II is warranted. Only the range-dependent case is discussed and the exact same procedure is followed in the range-independent case. The mean-square errors corresponding to different candidate ranges are shown in Fig. 15. The ambiguity of the least mean-square solution due to error in the arrival-time measurement $\sigma_{t}$ is also indicated in that figure. As we saw, the range giving the least mean-square error is $29.3 \mathrm{~km}$. Since the error $\sigma_{t}$ is $1.25 \times 10^{-2} \mathrm{~s}$, the explosive source range likely lies between the range at which the mean-square error is less than $\left(1.25 \times 10^{-2}\right)^{2}$. Assuming a uniform probability distribution in this ambiguity region $(27.8-30.8 \mathrm{~km})$, the uncertainty of the range estimate is characterized by a standard deviation $\sigma_{\mathrm{R}} 0.852 \mathrm{~km}$.

Finally, since our data are the inverse of the modal group velocity, the data variance can be approximated as the following in the same sense as the analysis for the group velocity:

$$
\sigma_{d}^{2}=\sigma_{v^{-1}}^{2} \approx\left(v^{G^{-1}} \frac{\sigma_{R}}{R}\right)^{2}
$$

which is suitable for both range-independent and range-dependent cases. Fig. 16 shows the variance of the inverse of the modal group velocity estimates in both cases. Using this value in (5) results in the depth-dependent variance in the estimate due to measurement inaccuracy, shown in Fig. 17 for both the range-independent and range-dependent inverses.

We note that the variance estimates in Fig. 17 are very small, in the order of $1 \mathrm{~m} / \mathrm{s}$. This is an artifact of our having already done much of the inverse "work" before doing the linear inverse, thus reducing the error. (The variance is particularly susceptible to this, whereas the resolution is largely unaffected.)

\section{DISCUSSION AND CONCLUSION}

In this paper, we have used an explosive "source of opportunity" to determine the sound speed profile of the sediments at the ASIAEX SCS experimental site. The analyses presented were generally standard ones, based on using the modal dispersion curves as data in a (primarily) linear inverse scheme. However, three new variants of the linear inverse were also presented in the Appendices: two using the mode cutoff frequency and the mode Airy phase as "special points" of the dispersion curve and a third performing a range-dependent linear inverse using the average group velocity and correct bathymetry. The first two methods allowed us to better described the subbottom layering structure and to ascertain a "basement" sound speed. Since our results also combined a priori data from chirp sonar and towed sources, it was also a "combined inverse," giving better resolution than could have been obtained from the broad-band inverse 


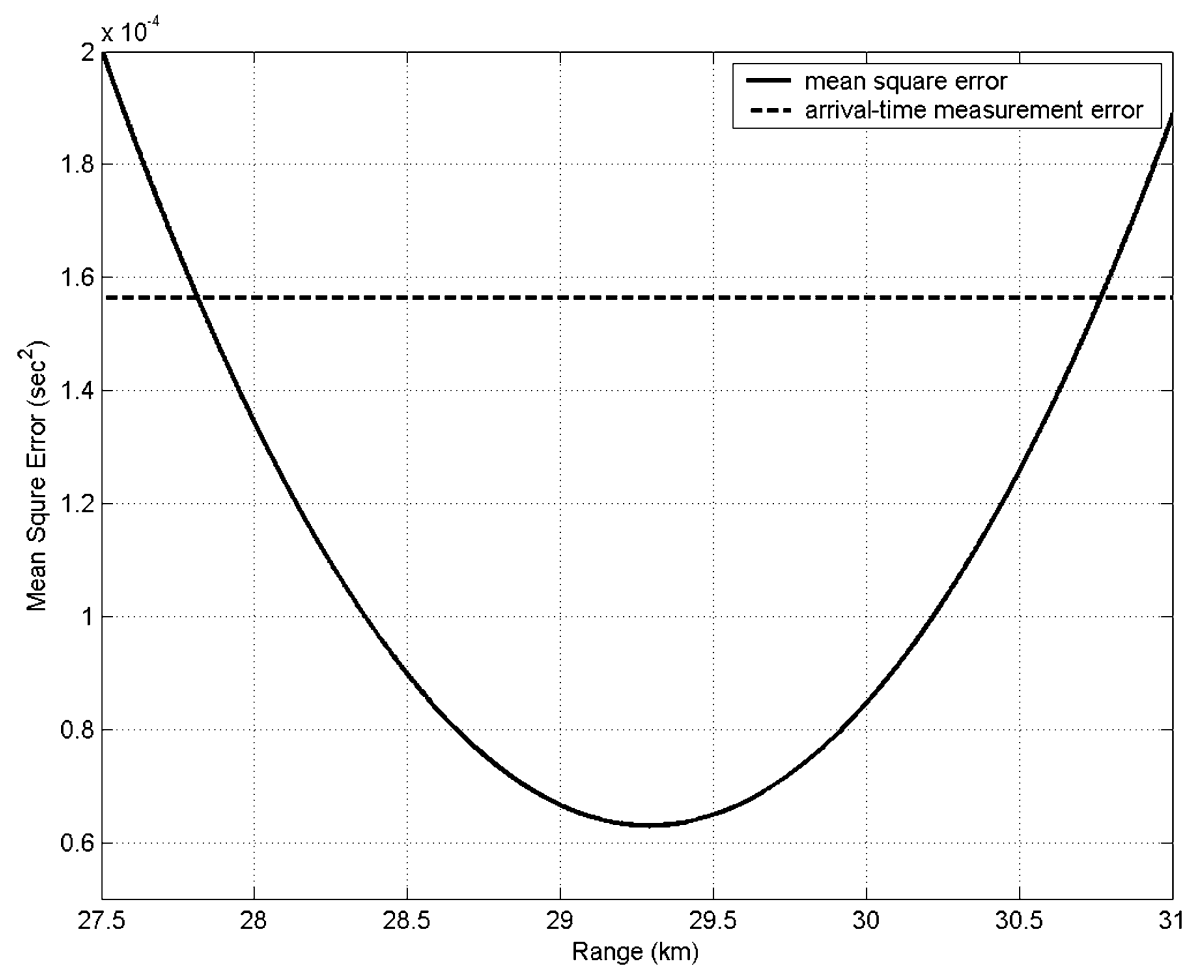

Fig. 15. Mean-square error corresponding to different ranges. The dashed line indicates the arrival-time measurement error.

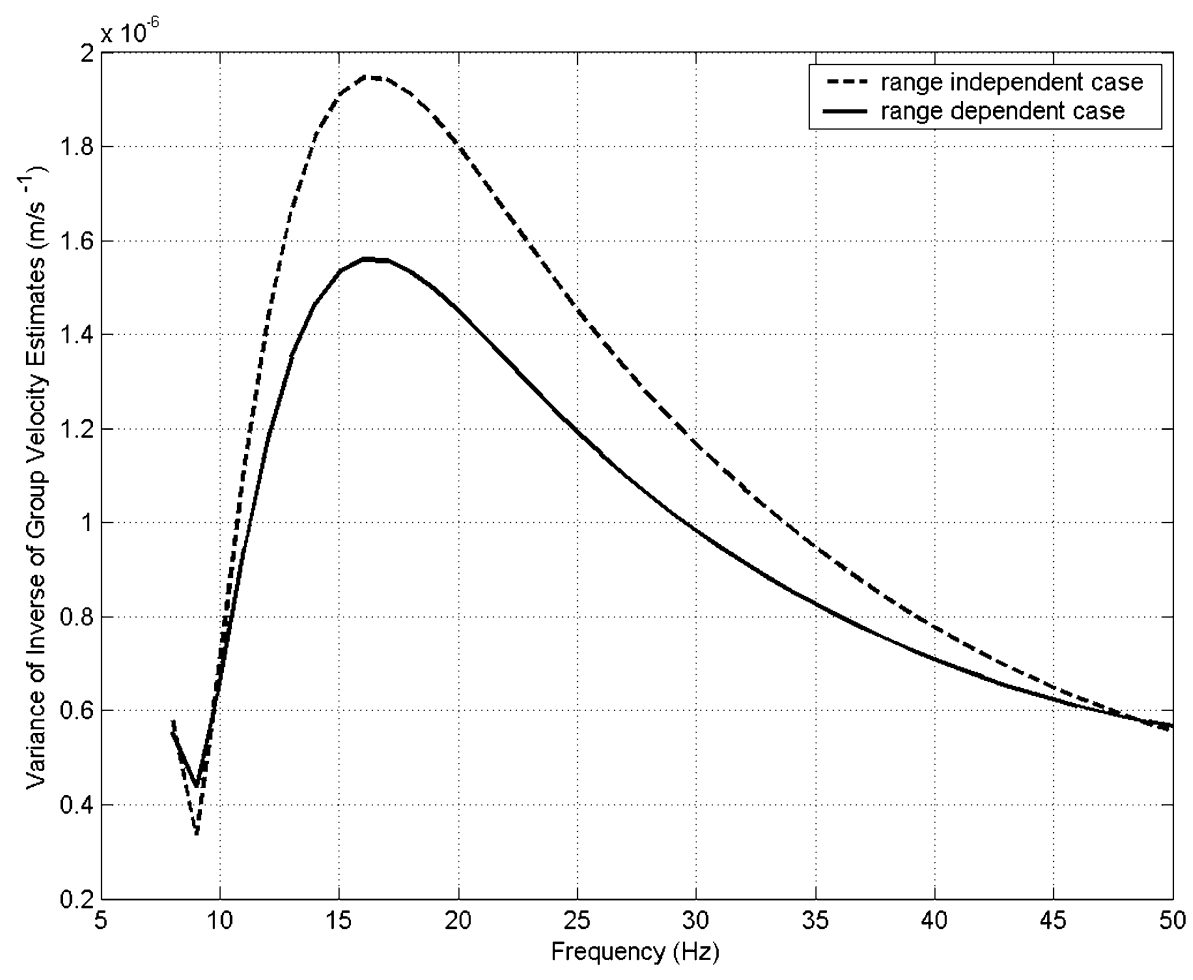

Fig. 16. Frequency-dependent variance of the inverse of modal group velocity estimates in both cases. In the range-dependent case, the modal group velocity estimate is a range-average velocity.

alone. Using the third new inverse variant, we have made an initial assault on the range-dependent broad-band linear inverse problem, though we would readily admit that there is much left to do on that topic.
The source of opportunity inverse was seen to be good in some aspects and suboptimal in others. The availability of a broad-band source with good low-frequency content was a major advantage, as it allowed us to probe deeper into the 


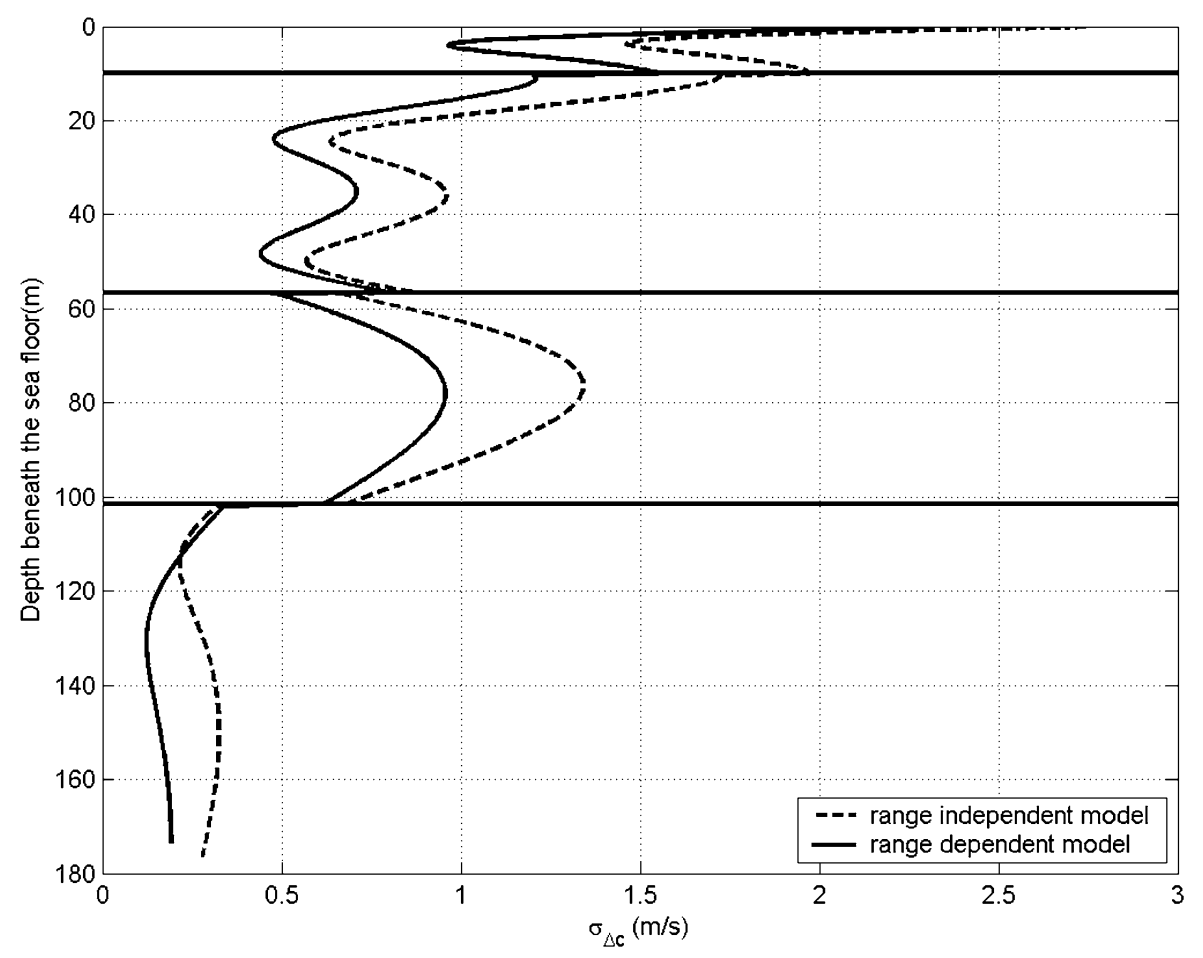

Fig. 17. Variance of bottom sound speed solution due to measurement inaccuracy $\sigma_{d}$ shown in Fig. 16. The dashed line is from the range-independent (receiver depth only) bathymetry and the solid line is from the range-dependent bathymetry.

bottom than our other sources permitted. We were able to estimate the range to the source using the higher frequency part of the dispersion curve. The lack of a source signature precluded obtaining an attenuation estimate and the lack of control of the source depth led to having fewer modes available to invert as we would have had were we able to set off the charges at a desired depth.

The possibilities for improving our results are being investigated. The use of the faint, incomplete, yet partially available mode one data, seen in the lower hydrophones may give better resolution, and we should examine how much improvement this small amount of extra data gives. We should examine the resolution and variance of the combined inverse, versus the broad-band data alone. The contribution of each type of data to the overall answer is an interesting study and one that we would like to pursue. Finally, the fully range-dependent broad-band adiabatic mode inverse needs to be further developed.

\section{APPENDIX A}

Perturbations of the Airy Phase Frequency AND the

Cutoff Frequency Due to a SMall Perturbation IN ENVIRONMENTAL SOUND SPEED

In this Appendix, new variants of standard perturbation formulations, based on 1) the Airy phase frequency and 2) the cutoff frequencies of acoustic modes in a range-independent waveguide, are presented.

Most of the perturbation theory work we will present here stems from older work on the perturbation of the horizontal wavenumber of the $n$th mode, $\Delta k_{n}$, due to the perturbed sound speed $\Delta c$ in a range-independent waveguide. This work was presented in, e.g., [14], which has the following equation:

$$
\begin{aligned}
\Delta k_{n}(\omega)=\frac{-1}{k_{n}^{(0)}(\omega)} & \int_{0}^{\infty} \rho^{(0)-1}(z) \\
& \times\left|Z_{n}^{(0)}(z, \omega)\right|^{2} \frac{\omega^{2}}{c^{(0) 3}(z)} \Delta c(z) d z
\end{aligned}
$$

where the superscript (0) indicates the unperturbed (background) environment, $Z_{n}$ is the modal function of $n$th mode, and $\rho$ and $\omega$ are the density and frequency, respectively. The frequency derivative of this equation gives the group velocity inverse formulae we used, and it also factors into the inverse schemes we will present.

\section{A. Airy Phase Frequency}

At the Airy phase frequency $\omega_{a}$, the first derivative of the $n$th modal group velocity, $v_{n}^{G}$, is equal to zero, i.e.,

$$
\left.\frac{d v_{n}^{G}}{d \omega}\right|_{\omega_{a}}=0
$$

After substituting $v_{n}^{G}=v_{n}^{G(0)}+\Delta v^{G}$ into the above equation, where $v_{n}^{G(0)}$ is the group velocity in the unperturbed environment, and $\Delta v^{G}$ is small change of group velocity due to the small perturbed sound speed $\Delta c$, we get

$$
\left.\frac{d v_{n}^{G(0)}}{d \omega}\right|_{\omega_{a}}+\left.\frac{d \Delta v^{G}}{d \omega}\right|_{\omega_{a}}=0
$$

Moreover, substituting the relation for the perturbed Airy phase $\omega_{a}=\omega_{a}^{(0)}+\Delta \omega_{a}$ into the first term of the LHS of the above formulation (keeping the second at $\omega_{a}$ ) and Taylor expanding 
around $\omega_{a}^{(0)}$ (ignoring the higher order terms), the following expression results:

$$
\left.\frac{d v_{n}^{G(0)}}{d \omega}\right|_{\omega_{a}^{(0)}}+\left.\frac{d^{2} v_{n}^{G(0)}}{d \omega^{2}}\right|_{\omega_{a}^{(0)}} \Delta \omega_{a}+\left.\frac{d \Delta v^{G}}{d \omega}\right|_{\omega_{a}}=0
$$

Since the first term of the above equation is equal to zero (by the definition of the Airy phase), the above equation can be rewritten as

$$
\left.\frac{d^{2} v_{n}^{G(0)}}{d \omega^{2}}\right|_{\omega_{a}^{(0)}} \Delta \omega_{a}+\left.\frac{d \Delta v^{G}}{d \omega}\right|_{\omega_{a}}=0
$$

which is an approximation of (A2) for small $\Delta \omega_{a}$.

We now look at the first term in (A3) in detail, relating it to the horizontal wavenumber $k_{n}$. Since $v_{n}^{G}=\left(d \omega / d k_{n}\right)=$ $\left(d k_{n} / d \omega\right)^{-1}$, the second derivative of $v_{n}^{G}$ can be expressed as

$$
\begin{array}{r}
\left.\frac{d^{2} v_{n}^{G(0)}}{d \omega^{2}}\right|_{\omega_{a}^{(0)}}=-\left.\left.\left.2 v_{n}^{G(0)}\right|_{\omega_{a}^{(0)}} \frac{d v_{n}^{G(0)}}{d \omega}\right|_{\omega_{a}^{(0)}} \frac{d^{2} k_{n}^{(0)}}{d \omega^{2}}\right|_{\omega_{a}^{(0)}} \\
-\left.\left.v_{n}^{G(0)^{2}}\right|_{\omega_{a}^{(0)}} \frac{d^{3} k_{n}^{(0)}}{d \omega^{3}}\right|_{\omega_{a}^{(0)}} .
\end{array}
$$

In addition, the first term of the RHS equals to zero, again, since $d v_{n}^{G(0)} / d \omega=0$ at the Airy phase, $\omega_{a}^{(0)}$. Therefore, the above can be simplified to

$$
\left.\frac{d^{2} v_{n}^{G(0)}}{d \omega^{2}}\right|_{\omega_{a}^{(0)}}=-\left.\left.v_{n}^{G(0)^{2}}\right|_{\omega_{a}^{(0)}} \frac{d^{3} k_{n}^{(0)}}{d \omega^{3}}\right|_{\omega_{a}^{(0)}}
$$

Next, we consider the second term of the (A3), relating it to a small change of the horizontal wavenumber $\Delta k$. Using $v_{n}^{G}=$ $v_{n}^{G(0)}+\Delta v^{G}$, we write

$$
\begin{aligned}
\Delta v^{G} & =v_{n}^{G}-v_{n}^{G(0)}=\frac{d \omega}{d k_{n}}-\frac{d \omega}{d k_{n}^{(0)}} \\
& =v_{n}^{G} v_{n}^{G(0)}\left(\frac{d k_{n}^{(0)}}{d \omega}-\frac{d k_{n}}{d \omega}\right)=-v_{n}^{G} v_{n}^{G(0)} \frac{d \Delta k}{d \omega} .
\end{aligned}
$$

Thus, the second term of (A3) can be expressed as

$$
\begin{aligned}
\left.\frac{d \Delta v^{G}}{d \omega}\right|_{\omega_{a}}= & \left.\left.\left(v_{n}^{G} v_{n}^{G(0)^{2}} \frac{d^{2} k_{n}^{(0)}}{d \omega^{2}}\right)\right|_{\omega_{a}} \frac{d \Delta k}{d \omega}\right|_{\omega_{a}} \\
& -\left.\left.\left(v_{n}^{G} v_{n}^{G(0)}\right)\right|_{\omega_{a}} \frac{d^{2} \Delta k}{d \omega^{2}}\right|_{\omega_{a}} .
\end{aligned}
$$

Finally, we may substitute (A4) and (A5) into (A3) to obtain

$$
\begin{aligned}
& \left.\left(v_{n}^{G(0)^{2}} \frac{d^{3} k_{n}^{(0)}}{d \omega^{3}}\right)\right|_{\omega_{a}^{(0)}} \Delta \omega_{a} \\
& \quad=\left.\left(v_{n}^{G} v_{n}^{G(0)^{2}} \frac{d^{2} k_{n}^{(0)}}{d \omega^{2}} \frac{d}{d \omega}-v_{n}^{G} v_{n}^{G(0)} \frac{d^{2}}{d \omega^{2}}\right)\right|_{\omega_{a}} \Delta k
\end{aligned}
$$

Furthermore, we can also obtain the perturbation due to a small perturbed sound speed $\Delta c$ via substituting $\Delta k$ in (A6) from
(A1). [We are looking at a single mode, so we do not explicitly show the index for mode $\mathrm{n}$ in $\Delta k$ in (A6).]

\section{B. Cutoff Frequency}

At the cutoff frequency $\omega_{c}$, there is an important condition that can be used to derive the perturbation of the cutoff frequency due to a small perturbation in sound speed, namely the modal group velocity equals the modal phase velocity $v^{P}$

$$
\left.v_{n}^{G}\right|_{\omega_{c}}=\left.v_{n}^{P}\right|_{\omega_{c}} .
$$

By definition, the above formation is equivalent to

$$
\left.\frac{d \omega}{d k}\right|_{\omega_{c}}=\left.\frac{\omega}{k}\right|_{\omega_{c}}
$$

which gives

$$
\left.k_{n}\right|_{\omega_{c}}=\left.\left(\omega \frac{d k_{n}}{d \omega}\right)\right|_{\omega_{c}}
$$

When we substitute the perturbed cutoff frequency $\omega_{c}=\omega_{c}^{(0)}+$ $\Delta \omega_{c}$ into (A7) and expand both sides via a Taylor expansion around the cutoff frequency for the unperturbed (background) waveguide $\omega_{c}^{(0)}$ (ignoring higher order terms again) we obtain

$$
\left.k_{n}\right|_{\omega_{c}}=\left.k_{n}\right|_{\omega_{c}^{(0)}+\Delta \omega_{c}}=\left.k_{n}\right|_{\omega_{c}^{(0)}}+\left.\frac{d k_{n}}{d \omega}\right|_{\omega_{c}^{(0)}} \Delta \omega_{c}
$$

for the LHS of (A7) and

$$
\begin{aligned}
\left.\left(\omega \frac{d k_{n}}{d \omega}\right)\right|_{\omega_{c}} & =\left.\left(\omega \frac{d k_{n}}{d \omega}\right)\right|_{\omega_{c}^{(0)}+\Delta \omega_{c}} \\
& =\left.\left(\omega \frac{d k_{n}}{d \omega}\right)\right|_{\omega_{c}^{(0)}}+\left.\left(\frac{d k_{n}}{d \omega}+\omega \frac{d^{2} k_{n}}{d \omega^{2}}\right)\right|_{\omega_{c}^{(0)}} \Delta \omega_{c}
\end{aligned}
$$

for the RHS.

Consequently, using (A8) and (A9), (A7) can be rewritten as

$$
\begin{aligned}
& \left.k_{n}\right|_{\omega_{c}^{(0)}}+\left.\frac{d k_{n}}{d \omega}\right|_{\omega_{c}^{(0)}} \Delta \omega_{c} \\
& \quad=\left.\left(\omega \frac{d k_{n}}{d \omega}\right)\right|_{\omega_{c}^{(0)}}+\left.\frac{d k_{n}}{d \omega}\right|_{\omega_{c}^{(0)}} \Delta \omega_{c}+\left.\left(\omega \frac{d^{2} k_{n}}{d \omega^{2}}\right)\right|_{\omega_{c}^{(0)}} \Delta \omega_{c} \cdot
\end{aligned}
$$

Canceling out the equal term on the both sides, we obtain

$$
\left.k_{n}\right|_{\omega_{c}^{(0)}}=\left.\left(\omega \frac{d k_{n}}{d \omega}\right)\right|_{\omega_{c}^{(0)}}+\left.\left(\omega \frac{d^{2} k_{n}}{d \omega^{2}}\right)\right|_{\omega_{c}^{(0)}} \Delta \omega_{c} .
$$

Finally, we substitute the perturbed horizontal wavenumber $k_{n}=k_{n}^{(0)}+\Delta k$ into (A10) to obtain the relationship between $\Delta \omega_{c}$ and $\Delta k$.

$$
\left.\left(\omega \frac{d^{2} k_{n}^{(0)}}{d \omega^{2}}\right)\right|_{\omega_{c}^{(0)}} \Delta \omega_{c}=\left.\left(1-\omega \frac{d}{d \omega}-\omega \Delta \omega_{c} \frac{d^{2}}{d \omega^{2}}\right)\right|_{\omega_{c}^{(0)}} \Delta k .
$$

Again, we can get the perturbation due to a small perturbed sound speed $\Delta c$ via substituting $\Delta k$ in (A11) from (A1). 


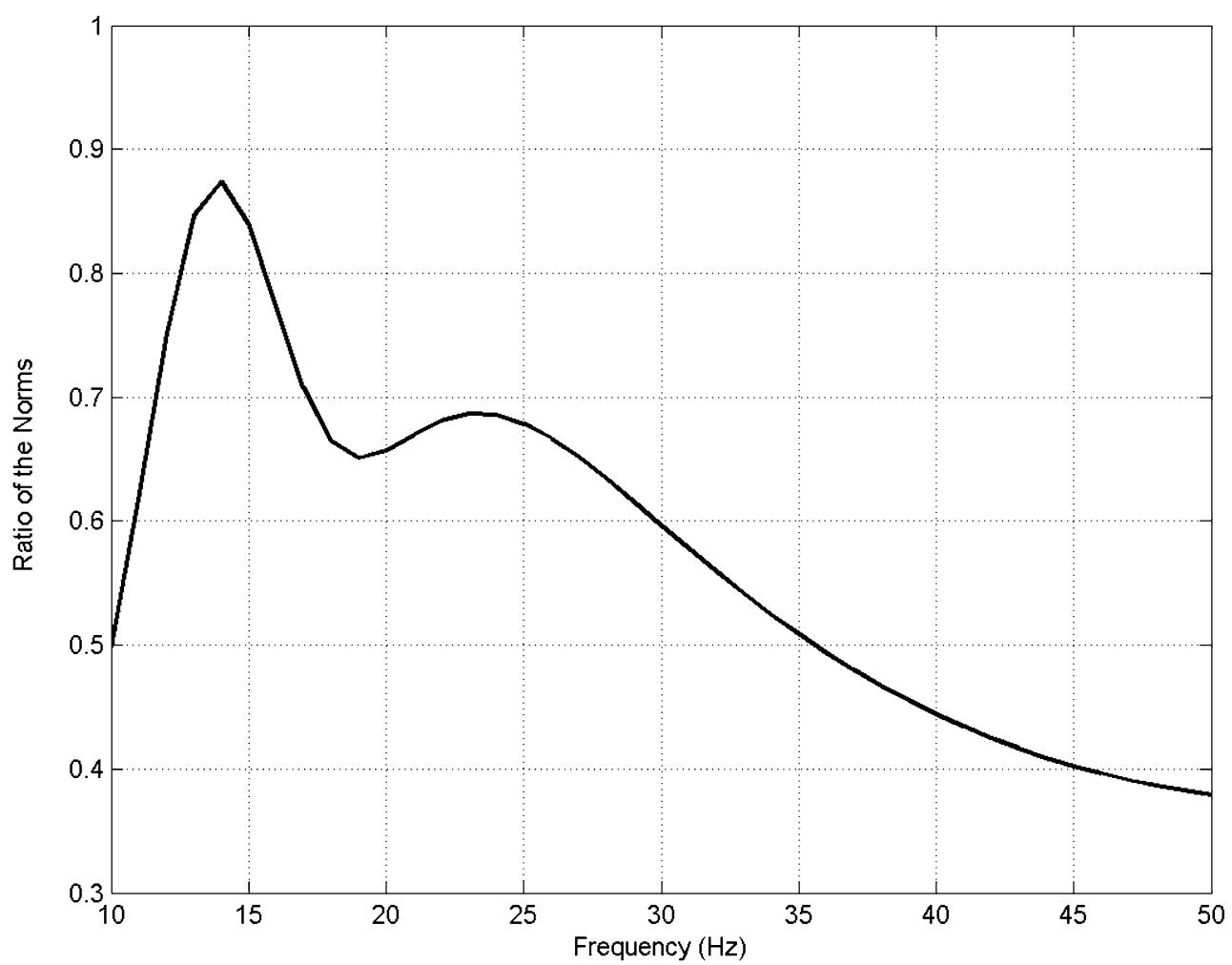

Fig. 18. Ratio of the norm of the standard deviation $D G_{n}^{(0)}(z, \omega)$ to the norm of the average $\bar{G}_{n}^{(0)}(z, \omega)$ in the range-dependent case presented in this paper.

\section{APPENDIX B}

\section{Perturbation of the GROUP Velocity} IN A RANGE-DEPENDENT MEDIUM

In a range-dependent environment, the average group velocity of an adiabatic mode between source and receiver can be expressed as

$$
\bar{v}_{n}^{G}(\omega)=R / \frac{d}{d \omega} \int_{R} k_{n}(r, \omega) d r
$$

where $\mathbf{R}$ is the distance between source and receiver. Therefore, the difference of the inverse of the average group velocity from the background model can be related to the difference of the horizontal wavenumber in the following way

$$
\Delta \frac{1}{\bar{v}_{n}^{G}}(\omega)=\frac{1}{R} \int_{R} \frac{d \Delta k_{n}(r, \omega)}{d \omega} d r .
$$

The integrand on the RHS of the last equation is calculated using (A1). Thus, the average modal group velocity can be expressed as follows:

$$
\Delta \frac{1}{\bar{v}_{n}^{G}}(\omega)=\frac{1}{R} \int_{R} \int_{0}^{\infty} G_{n}^{(0)}(r, z, \omega) \Delta c(r, z) d z d r
$$

where $G_{n}^{(0)}(r, z, \omega)$ is the so-called "transform kernel" that is now range-dependent. This kernel governs the relationship between the difference of sound speed from the background and the difference of the inverse of the average group velocity from the background.

There is no way to solve for a range-dependent unknown $\Delta c(r, z)$ from data at only one range. However, we may decompose the range-dependent parameters in the above integral into an average term over the range and a variation from the average, as follows:

$$
\begin{aligned}
G_{n}^{(0)}(r, z, \omega) & =\bar{G}_{n}^{(0)}(z, \omega)+\delta G_{n}^{(0)}(r, z, \omega) \\
\Delta c(r, z) & =\overline{\Delta c}(z)+\delta \Delta c(r, z) .
\end{aligned}
$$

Substituting these expansions around the average into the perturbation equations leads to the following formulation

$$
\begin{aligned}
\Delta \frac{1}{\bar{v}_{n}^{G}}(\omega) & =\int_{0}^{\infty} \bar{G}_{n}^{(0)}(z, \omega) \overline{\Delta c}(z) d z \\
+ & \int_{0}^{\infty}\left[\frac{1}{R} \int_{R} \delta G_{n}^{(0)}(r, z, \omega) \delta \Delta c(r, z) d r\right] d z
\end{aligned}
$$

If the last integral is small enough to be ignored, the average unknown $\overline{\Delta c}(z)$ can be estimated well via this linear inverse formula. Whether or not the second integral is small or not is an important point, and we address it next.

In order to understand the residual term, we can bound the second integral using Schwartz's inequality, i.e.,

$$
\begin{aligned}
& {\left[\frac{1}{R} \int_{R} \delta G_{n}^{(0)}(r, z, \omega) \delta \Delta c(r, z) d r\right]^{2}} \\
& \quad \leq \frac{1}{R} \int_{R}\left|\delta G_{n}^{(0)}(r, z, \omega)\right|^{2} d r \times \frac{1}{R} \int_{R}|\delta \Delta c(r, z)|^{2} d r
\end{aligned}
$$

This inequality implies that two statistics are useful to describe the error from the residual term in the (A14). They are the standard deviation of the transform kernel $D G_{n}^{(0)}(z, \omega)$ and the standard deviation of the sound speed difference $D \delta \Delta c(z)$. These can be expressed as

$$
D G_{n}^{(0)}(z, \omega)=\sqrt{\frac{1}{R} \int_{R}\left|\delta G_{n}^{(0)}(r, z, \omega)\right|^{2} d r}
$$




$$
D \delta \Delta c(z)=\sqrt{\frac{1}{R} \int_{R}|\delta \Delta c(r, z)|^{2} d r}
$$

Thus, the error-bound to (A14) can be written as

or

$$
\begin{aligned}
& \pm \int_{0}^{\infty} \sqrt{\frac{1}{R} \int_{R}\left|\delta G_{n}^{(0)}(r, z, \omega)\right|^{2} d r} \\
& \times \sqrt{\frac{1}{R} \int_{R}|\delta \Delta c(r, z)|^{2} d r} d z
\end{aligned}
$$

$$
\pm \int_{0}^{\infty} D G_{n}^{(0)}(z, \omega) \cdot D \delta \Delta c(z) d z
$$

For the case presented in this paper, the ratio of the norm of the standard deviation of the transform kernel to the norm of the average transform kernel (see Fig. 18) provides some insight into this error, if we assume for now the spatial variation of the sound-speed solution $D \delta \Delta c(z)$ is of the order of the $\overline{\Delta c}(z)$. (We are somewhat forced in to this latter, bounding assumption, as we have no way to directly calculate $D \delta \Delta c(z)$.) It is seen that the residual term in (A14) is smaller than the first term, though not greatly so, and becomes less important as frequency increases. We also note that our residual term error seems to be greatest at the Airy phase frequency, for reasons we do not currently understand.

\section{ACKNOWLEDGMENT}

First and foremost, the authors must thank the dynamite fisherman, whose source made this study possible. The authors hope he caught many fish that day! Second, the authors must thank all of their sponsors, colleagues, and students for their support, collaboration, hard work, and sense of humor, without which ASIAEX would not have succeeded. In particular, they will single out the ASIAEX coordinators, whose "behind the scenes" efforts cleared the path for the experiment, the captain and crew of the Fisheries Researcher I, which deployed and recovered their acoustic array, their super engineering and buoy deployment teams, which got them the data, Potty and Miller for their detailed comments on this manuscript and their wavelet calculations, and S. Wolf of NRL for his HLA localizations. This is WHOI Contribution Number 11009.

\section{REFERENCES}

[1] A. Turgut et al., "Inversion of range-dependent geoacoustic properties in South China Sea ASIAEx01 experimental site," J. Acoust. Soc. Amer., vol. 113, p. $2218,2003$.

[2] S. Schock, "A method for estimating the physical and acoustical properties of the seabed using chirp sonar data," IEEE J. Oceanic Eng., vol. 29 , pp. 1200-1217, Oct. 2004.

[3] , "Remote prediction of physical and acoustical sediment properties in the South China Sea using chirp sonar data and the Biot model," IEEE J. Oceanic Eng., vol. 29, pp. 1218-1230, Oct. 2004.

[4] L. Bartek and C. S. Liu, private communication.

[5] A. Newhall et al., "Preliminary acoustic and oceanographic observations from the ASIAEX 2001 South China Sea experiment," Woods Hole Oceanographic Institution, Tech. Rep., WHOI-2001-12, 2001.

[6] J. Potter, private communication.

[7] A. V. Oppenheim et al., Discrete-Time Signal Processing. Englewood Cliffs, NJ: Prentice-Hall, 1999.

[8] N. R. Chapman et al., "Source levels of shallow explosive charges," $J$. Acoust. Soc. Amer., vol. 84, pp. 697-702, 1988.
[9] M. B. Porter, "The KRAKEN normal mode program," SACLANT Undersea Research Centre, La Spezia, Italy, Rep. SM-245, 1991.

[10] T. H. Schroeder, "Horizontal linear array sensor localization and preliminary coherence measurements from the 2001 ASIAEX South China Sea experiment," Master's thesis, MIT/WHOI, 2002.

[11] S. Wolf, private communication.

[12] E. L. Hamilton, "Sound velocity-density relations in sea-floor sediments and rocks," J. Acoust. Soc. Amer, vol. 63, pp. 1366-1377, 1978.

[13] — "Geoacoustic modeling of the sea floor," J. Acoust. Soc. Amer. vol. 68, pp. 1313-1340, 1980

[14] S. D. Rajan et al., "Perturbative inversion methods for obtaining bottom geoacoustic parameters in shallow water," J. Acoust. Soc. Amer., vol. 82, pp. 998-1017, 1988.

[15] J. F. Lynch et al., "A comparison of broadband and narrow-band modal inversions for bottom geoacoustic properties at a site near Corpus Christi, Texas," J. Acoust. Soc. Amer., vol. 89, pp. 648-665, 1988.

[16] C. Wunsch, The Ocean Circulation Inverse Problem. Cambridge, U.K.: Cambridge University Press, 1996.

[17] W. J. Emery and R. E. Thomson, Data Analysis Methods in Physical Oceanography. New York: Pergamon, 1997.

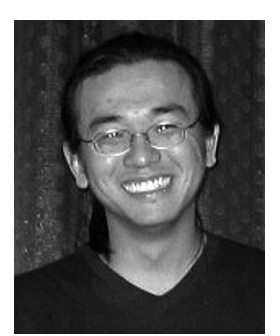

Ying-Tsong Lin received the B.S. degree in hydraulic and ocean engineering from National Cheng Kung University, Taiwan, in 1996, the M.S. degree in naval architecture and ocean engineering and the $\mathrm{Ph} . \mathrm{D}$. degree in engineering science and ocean engineering both from the National Taiwan University, Taiwan, in 1998 and 2004, respectively.

From 2002 to 2003, he was a Guest Investigator with the Applied Ocean Physics and Engineering Department, Woods Hole Oceanographic Institution (WHOI), Woods Hole, MA. After receiving the Ph.D. degree, he began a Postdoctoral appointment of a half-year at Underwater Acoustics Laboratory, National Taiwan University, and also received a Postdoctoral award from the WHOI. Currently, he is a Postdoctoral Scholar with the WHOI. His research interests include acoustic propagation modeling, geoacoustic inversion, and source localization.

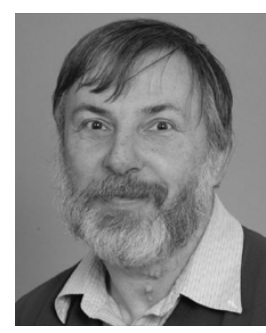

James F. Lynch (M'96-SM'03) was born in Jersey City, NJ, on June 3, 1950. He received the B.S. degree in physics from Stevens Institute of Technology, Hoboken, NJ, in 1972 and the Ph.D. degree in physics from the University of Texas, Austin, in 1978.

He was with the Applied Research Laboratories, University of Texas at Austin (ARL/UT) from 1978 to 1981, after which he joined the scientific staff at the Woods Hole Oceanographic Institution (WHOI), Woods Hole, MA. He has been with WHOI since then and currently holds the position of Senior Scientist in the Applied Ocean Physics and Engineering Department. His research specialty areas are ocean acoustics and acoustical oceanography, but he also greatly enjoys occasional forays into physical oceanography, marine geology, and marine biology.

Dr. Lynch is a Fellow of the Acoustical Society of America and Editor-inChief of the IEEE JOURNAL OF OCEANIC ENGINEERING.

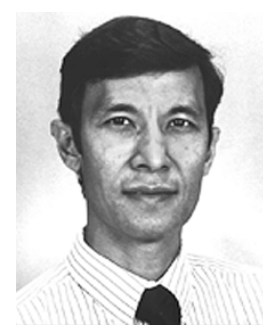

Nick Chotiros received the B.A. degree in engineering from Cambridge University, England, in 1973, and the Ph.D. degree in electronic and electrical engineering from University of Birmingham, England, in 1981.

From 1974 to 1982, he was a Lecturer at the University of Birmingham. Since 1982, he has been a Research Associate at the Applied Research Laboratories, The University of Texas, Austin. He worked on a number of projects in underwater acoustics while at both universities.

Dr. Chotiros is a Member of the Acoustical Society of America. Currently, he is serving as a Program Officer at the Office of Naval Research. 


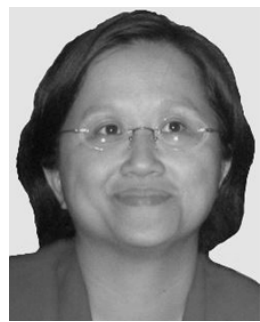

Chi-Fang Chen was born in Taipei, Taiwan, in 1959. She received the B.S. degree in naval architecture from National Taiwan University, Taipei, Taiwan, in 1981 and the M.S. and Ph.D. degrees in ocean engineering from Massachusetts Institute of Technology, Cambridge, in 1984 and 1990, respectively.

She then returned to Taiwan and became an Associate Professor with the National Taiwan University. Since 2002, she has been a full Professor with the Department of Engineering Science and Ocean Engineering, National Taiwan University. She has devoted her research to underwater acoustics, particularly 3-D acoustic propagation and acoustic inversion.

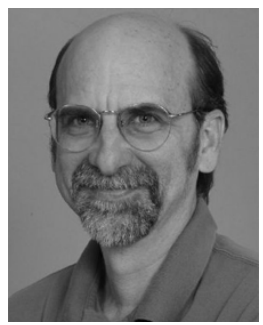

Arthur Newhall received the B.S. degree in mathematics from the University of Maine at Orono in 1985.

$\mathrm{He}$ is a Research Specialist with the Applied Ocean Physics and Engineering Department, Woods Hole Oceanographic Institution, Woods Hole, MA. His current interests include ocean acoustic propagation modeling, acoustical oceanography, ocean acoustic tomography, and software engineering.

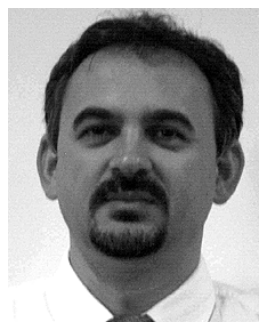

Altan Turgut received the Ph.D. degree in ocean engineering and applied marine physics from the University of Miami, Coral Gables, FL, in 1990.

Since 1995, he has been a Research Physicist in the Acoustics Division, Naval Research Laboratory, Washington, DC. His main research interests are acoustic wave propagation in shallow water and geophysical inversion techniques, which include in situ measurements of deterministic and stochastic properties of marine sediments and their effects on acoustic wave propagation in ocean waveguides.

Dr. Turgut is a Member of ASA and AGU.

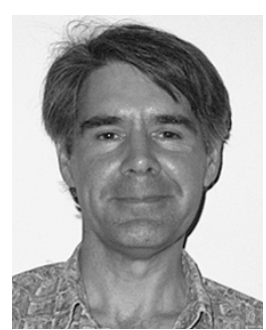

Steven G. Schock (M'86) was born in Warwick, R.I., in 1956. He received the B.S. degree from the U.S. Naval Academy, Annapolis, MD, in 1979 and the Ph.D. degree from University of Rhode Island, Narragansett, in 1989, both in ocean engineering.

After receiving the B.S. degree, he entered the U.S. Navy Nuclear Power Program and served as Weapons Officer on USS Dallas (SSN-700). Currently, he is a Professor of Ocean Engineering at Florida Atlantic University, Boca Raton, where he has been conducting research and teaching since 1989. His research interests include bottom interacting acoustics, sediment classification, sea-bed and buried-object imaging, and sonar design.

Dr. Schock is a Member of the Acoustical Society of America and the Marine Technology Society.

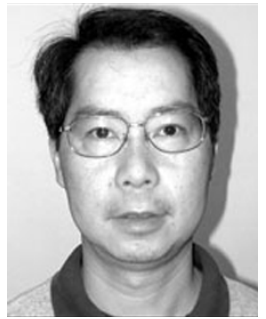

Ching-Sang Chiu received the Ph.D. degree from the Massachusetts Institute of Technology (MIT), Cambridge, and Woods Hole Oceanographic Institution (WHOI), Woods Hole, MA, joint program in 1985.

$\mathrm{He}$ is Professor of Oceanography at the Naval Postgraduate School, Washington, DC. His current research interests include shallow-water acoustics (particularly volume variability effects in shelfbreak regions, coastal ocean acoustic tomography, and passive detection) and censusing of marine mammal vocalizations.

Dr. Chiu is a Fellow of the Acoustical Society of America.

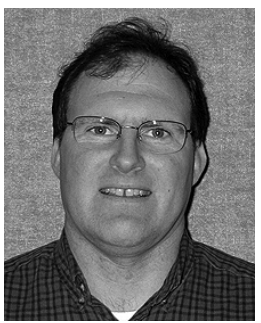

Louis R. Bartek received the B.S. degree from Pennsylvania State University, State College, and the $\mathrm{Ph} . \mathrm{D}$. degree from Rice University, Houston, TX, both in geology.

He currently is an Associate Professor in the Department of Geological Sciences, University of North Carolina, Chapel Hill. His research interests include the impact of climatic change (i.e., sea level, sediment supply) on the geologic record of continental margins, establishing stochastic models of spatial distribution of physical attributes on continental margins subjected to various environmental boundary conditions, and improving understanding of proximal record of climate change on Antarctic continental margin with proxy records from the deep sea.

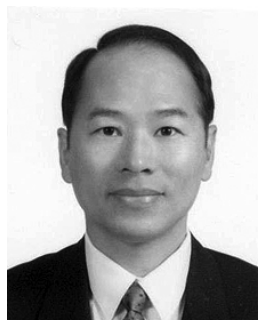

Char-Shine Liu received the B.S. degree in geophysics from National Central University, Taiwan, in 1995, and the M.S. and Ph.D. degrees in earth sciences from Scripps Institution of Oceanography, University of California, San Diego, in 1980 and 1983 , respectively. He finished a two-year postdoctoral work at Cornell University, Ithaca, NY, in 1985.

He moved to Standard Oil Company, Texas, in 1985, where he worked for three years in the research center. He became an Associate Professor at the Institute of Oceanography, National Taiwan University, Taiwan, in 1988. He has been a Professor at that Institute since 1994. He also served as the Deputy Director of the National Center for Ocean Research from 2002 to 2003 and is currently the Director. His research interests include seismic reflection exploration, sea floor surveying, and gas hydrate investigation.

Prof. Liu is a Member of the American Geophysical Union, Chinese Geosciences Union (Taiwan), Geological Society (Taipei), and Chinese Undersea Technology Association (Taiwan). He is a Chairman of the SCOR Taiwan Committee. 